\title{
A Causal Framework for Cross-Cultural Generalizability
}

\author{
Dominik Deffner ${ }^{1,2,3^{*}}$, Julia M. Rohrer ${ }^{4} \&$ Richard McElreath $^{1}$ \\ ${ }^{1}$ Department of Human Behavior, Ecology and Culture, Max Planck Institute for Evolutionary An- \\ thropology, Leipzig, Germany \\ ${ }^{2}$ Science of Intelligence Excellence Cluster, Technical University Berlin, Berlin, Germany \\ ${ }^{3}$ Center for Adaptive Rationality, Max Planck Institute for Human Development, Berlin, Germany \\ ${ }^{4}$ Department of Psychology, Leipzig University, Leipzig, Germany \\ ${ }^{*}$ Corresponding author: deffner@mpib-berlin.mpg.de
}

\begin{abstract}
Behavioral researchers increasingly recognize the need for more diverse samples that capture the breadth of human experience. Current attempts to establish generalizability across populations focus on threats to validity, constraints on generalization and the accumulation of large cross-cultural datasets. But for continued progress, we also require a framework that lets us determine which inferences can be drawn and how to make informative cross-cultural comparisons. We describe a generative causal modeling framework and outline simple graphical criteria to derive analytic strategies and implied generalizations. Using both simulated and real data, we demonstrate how to project and compare estimates across populations and further show how to formally represent measurement (in-)equivalence across societies. We conclude with a discussion of how a formal framework for generalizability can assist researchers in designing more informative cross-cultural studies and thus provides a more solid foundation for cumulative and generalizable behavioral research.
\end{abstract}

Keywords: Cross-cultural research, generalizability, WEIRD samples problem, causal inference, poststratification.

\section{INTRODUCTION}

The behavioral and social sciences have been criticized for relying excessively on WEIRD samples in which most participants are Western, educated, and from industrialized, rich, and democratic countries (Henrich et al. 2010, Apicella et al. 2020, Henrich 2020, Muthukrishna

5 et al. 2020). Research has established substantial cross-cultural variation in key psychological domains, such as thinking styles (e.g. Masuda and Nisbett 2001, Nisbett and Miyamoto 2005), economic preferences (e.g. Gächter and Schulz 2016, Falk et al. 2018), personality structure (e.g. Smaldino et al. 2019) and moral judgements (e.g. Curtin et al. 2020, Awad et al. 2020), and furthermore demonstrated that WEIRD subjects often represent outliers 10 among present-day societies (Apicella et al. 2020). These findings make it clear that broad, unqualified generalizations about human psychology based on WEIRD samples alone are rarely justified.

Fortunately, behavioral scientists increasingly acknowledge the problem. Cross-cultural psychologists and anthropologists are making progress in documenting variation in psycho15 logical phenomena (Apicella et al. 2020). In addition to long-term fieldwork and experimental comparisons across societies, large-scale collaborative projects have started compiling extensive datasets addressing cross-cultural variation and commonality in domains such as music (Mehr et al. 2019), social perception (Jones et al. 2021), and economic (Henrich et al. 2001) and moral decision making (Awad et al. 2018). Accompanying the surge in 20 cross-cultural studies, researchers increasingly consider the historical and political contexts of their work, as well as its ethical ramifications (e.g. Broesch et al. 2020, Urassa et al. 2021, Clancy and Davis 2019, Ghai 2021). 
New data bring new problems. How can valid comparisons and conclusions be derived from cross-cultural samples? Just as there are many ways to misinterpret data from a 25 single society, there are even more ways to misinterpret differences or similarities between societies. In each case, one must first generalize from each sample to each population before valid comparisons can be made between populations. This is a generalizability problem on a global scale.

Methodologists have long discussed the importance of generalizability or "external validity" and its relationship to other kinds of validity (internal, statistical conclusion and construct validity) (e.g. Campbell 1957, Cock and Campbell 1976, Berkowitz and Donnerstein 1982, Calder et al. 1983, Winer 1999). Researchers trained in psychology and other behavioral sciences may be familiar with catalogs of threats to validity that describe prototypical problematic situations (Matthay and Glymour 2020). These lists can grow

35 rapidly. For external validity alone, Shadish et al. (2002) distinguish five types of threats that include interactions of the causal relationship of interest with specific units, settings, mediators, outcomes and treatment variations.

Because of these threats, there have been reasonable calls for constraint. Yarkoni (2020), for instance, has argued that poor alignment between verbal hypotheses and quantitative inference lies at the heart of many of psychology's problems; narrow and seemingly arbitrary operationalizations of broad constructs invalidate the intended generalizations. As a remedy against (often implicit) unwarranted generalizations, researchers have proposed to add mandatory "Constraints on generality (COG)" statements to all empirical papers (Simons et al. 2017, Tiokhin et al. 2019). By specifying sample characteristics and assessing

45 its representativeness of wider populations, such COG statements are meant to discipline authors to explicitly state intended generalizations and thereby improve transparency.

These steps towards a more global and generalizable science are overdue. However, under the current framework - with its emphasis on threats to validity, constraints and the accumulation of cross-cultural samples - only limited progress can be made. Lists of threats 50 are devices that raise awareness of inferential problems, but they are not also solutions. They do not spell out which inferences are warranted, and under which assumptions. This leads to the impression that any claim which goes beyond the precise operationalization, population, and historical context of a study overgeneralizes.

From this perspective, it is understandable that researchers are eager to collect rich data sets just to describe what is "out there" (e.g. Rozin 2001, Barrett 2020). But even this is not possible without an explicit framework that licenses generalization. In a crosscultural context, even "mere description" and simple comparisons rely upon usually implicit assumptions that permit moving from sample to population and across populations.

Threats and constraints forbid inference; we require a framework that also licenses inference. Such a framework would inform researchers about the assumptions underlying potential generalizations, assist them in the design of empirical studies, and show them how to construct appropriate statistical procedures. Such a framework already exists and has sparked a "causal revolution" (Pearl 2018) in computer science and machine learning, but it is not a standard part of training in the behavioral and social sciences. This

65 framework depends upon transparent, generative models of research. One key idea is that generalizability does not depend on the presence of sample differences per se, nor on raw statistical associations. The conditions that license generalization and comparison to other populations depend on the causal relations between variables and the exact mechanisms by which populations differ.

70 Many cross-cultural scientists already pay close attention to concerns of causal inference and comparison, without use of a formal framework (e.g. Pollet et al. 2014, Norenzayan and 
Heine 2005). For these researchers, a formalized framework can provide a vocabulary to articulate their concerns and work toward solutions in a more systematic manner.

For instance, many researchers will share the intuition that the demographic breakdown 75 and other relevant factors should be somehow standardized across groups to eliminate potential confounds. A standard approach to dealing with such threats to validity and crosscultural comparison is to condition on (i.e., adjust or "control" for) any potential confounds such as age, income or methodological differences by, for example, including such variables as predictors in multiple regression (conditioning on a variable means to analyze the values 80 of other variables for a given, constant, value of the conditioned variable). But it is not enough to mechanically control for a set of variables that may vary across populations. One reason is that not all controls are good-adding variables can bias inference as much as it can correct it (Cinelli et al. 2020). An important example is "collider bias" where a spurious association between two variables arises as we include a third variable, which is jointly caused by those variables. As we will see below, which variables act as confounds depends on the assumed causal structure and the specific research question. A formal generative framework lets us logically deduce which variables we should - and should not - control for in any cross-cultural comparison. Going beyond the question of which variables to include, it also helps us derive the appropriate statistical estimates that actually align with the scientific goal at hand. Coefficients and parameters themselves are valid measures of difference or causal effect in only the simplest models (Morgan and Winship 2015, Rohrer and Arslan 2021). Knowing a cause means that we can predict the consequences of an intervention (Woodward 2005, Morgan and Winship 2015, Athey and Imbens 2016, Greene 2000, Asteriou and Hall 2015) and most causal questions require the construction of "marginal" effects, where we average the effect of interest over the influence of all other important variables to find out how an dependent variable would change if we intervened on the independent variable. Such "poststratification", that is, re-weighting of model estimates to answer specific causal questions, only becomes more complicated when comparisons are made between societies (Oganisian and Roy 2021).

100 In short, there is no universally valid procedure for cross-cultural inference. For each inferential problem, we have to start with a generative causal model that lets us determine the role variables play in the analysis, and how to construct statistical summaries that are logically derived from transparent research goals.

In the rest of this paper, we outline a formal framework for cross-cultural generalizability based on recent advances in the fields of causal inference and data fusion (Pearl and Bareinboim 2014, Pearl 2015, Bareinboim and Pearl 2016, Lundberg et al. 2021). We apply these established formal tools to commonplace questions in cross-cultural research: (1) description of cultural variation, (2) comparison of causal effects identified through experiments and (3) measurement (in-)equivalence of latent constructs. To help researchers adopt this

110 approach, we provide example causal diagrams and statistical analyses using simulated and real-world cross-cultural data. Finally, we discuss how our framework can assist researchers in planning targeted cross-cultural comparisons and designing more informative studies.

\section{A Causal Framework}

A causal framework for cross-cultural research requires us to state (1) what we want to 115 know, that is, the estimand, (2) a generative model of the evidence, that is, a causal model of how the observed data came into existence, (3) a generative model of how populations may differ, and (4) a tailored estimation strategy that allows us to learn from data. We first develop these requirements in general terms. In later sections, we discuss specific examples. 
2.1. Theoretical and Empirical Estimands. The starting point for any empirical analysis is the theoretical estimand. This is the target of the analysis derived from theory (see Lundberg et al. 2021, for an excellent introduction). A theoretical estimand consists of a unit-specific quantity as well as a target population. It is defined outside of any statistical model - not in terms of, for example, regression coefficients. We may simply be interested in the mean of a variable in a certain population (e.g., probability that individual $i$ chooses the prosocial option in a dictator game, averaged over all individuals $i$ in target population); or we may be interested in the average treatment effect of some independent variable on an outcome in a certain population (e.g., effect of norm prime on probability that individual $i$ chooses prosocial option, averaged over all individuals $i$ in target population; examples inspired by House et al. (2020), see below).

130 Once the theoretical estimand is set, we need to link it to an empirical estimand. While the theoretical estimand might contain unobservable quantities such as counterfactuals ("what would have been true under different circumstances?"), the empirical estimand is defined solely in terms of observed data. We cannot observe the average probability of prosocial choice for the whole population; however, we can try to estimate it from a sample. We also cannot observe individual-level causal effects, but we may estimate their average by considering observed differences between randomized experimental conditions.

In the context of cross-cultural research, the distinction between theoretical and empirical estimands encourages researchers to explicitly spell out assumptions about how theoretical constructs (e.g., prosociality) can be operationalized in comparable ways across societies 140 ("construct validity"). This issue of measurement (in-)equivalence and bias is further discussed in section 3.3.

2.2. Directed Acyclic Graphs (DAGs). A valid link between theoretical and empirical estimand requires causal assumptions. Generative models embody causal assumptions, and there are many forms these models can take. One popular approach are Directed 145 Acyclic Graphs (DAGs). This approach is accessible thanks to its graphical nature; it can be used to develop an intuitive understanding of inferential obstacles; and it can alert researchers to inferential opportunities they had not considered. There are other suitable ways to spell out assumptions (e.g., psychological process models (Farrell and Lewandowsky 2018)), and not all generative models can be formalized with the help of DAGs. But DAGs

150 provide a pragmatic starting point and can be extended to include commonplace issues like measurement error and missing data (see McElreath 2020, chapter 15).

Multiple comprehensive yet accessible introductions to DAGs are available (Elwert 2013, Pearl et al. 2016, Rohrer 2018, Pearl and Mackenzie 2018), thus, we will focus only on the essentials. In DAGs, nodes represent variables and arrows represent causal effects. For 155 example, Figure 1a captures a set of assumptions regarding the associations between age, prosociality, reputation, and the outcome of a dictator game. The arrows indicate causal effects that may take any functional form, which includes any possible interaction between variables that jointly affect another variable. Individual paths can be identified by travelling along the arrows connecting any pair of variables. These paths can be broken down into fundamental structures (see Box 1) that determine whether a given path transmits an association between variables, and whether the association is causal or non-causal.

Suppose we were interested in the causal influence of prosociality on dictator game choice in the population from which we randomly drew our sample. If we are willing to assume that the depicted DAG is a causal DAG - which means that it includes all common causes of any 165 pair of variables (Elwert 2013) - we are able to algorithmically derive which variables need to be "conditioned" (see Box 1) on to identify the causal effect of interest. In this particular example, the answer is easy. There is only one open non-causal path (see Box 1) between 
prosociality and dictator game choice: Prosociality $\leftarrow$ Age $\rightarrow$ Dictator game choice. Since age is a common cause of prosociality and dictator game choice, some of the association between both variables is due to this non-causal path. This path can be blocked by conditioning on age (again see Box 1). Thus, we have discovered a way to link the theoretical estimand (the effect of prosociality on dictator game choice in our population) to an actual empirical estimand that we can estimate from observable data. If, instead, our theoretical estimand was the effect of the norm prime, no conditioning would be necessary for causal identification: as the norm prime has been randomized, no backdoor paths can exist (no arrows point into the randomized variable). The simple mean difference between experimental groups would be an empirical estimand that corresponds to the theoretical estimand under the assumptions embodied in the DAG (taking into account other variables that influence the outcome may still be helpful to improve precision).

\section{Box 1: Elementary Causal Structures}

Any path connecting two variables can be broken down into three fundamental causal structures: chains, forks, and inverted forks (Elwert 2013, Rohrer 2018).

Chains: $\mathrm{X} \rightarrow \mathrm{M} \rightarrow \mathrm{Y}$. The chain transmits a causal association between $\mathrm{X}$ and Y. If we condition on M (the mediator; e.g., through statistical adjustment, sample stratification, or by design), the transmission of the association is blocked.

Forks: $\mathrm{X} \leftarrow \mathrm{C} \rightarrow \mathrm{Y}$. The fork transmits a non-causal association between $\mathrm{X}$ and $\mathrm{Y}$. If we condition on $\mathrm{C}$ (the confounder), the transmission of the association is blocked.

Inverted forks: $\mathrm{X} \rightarrow \mathrm{L} \leftarrow \mathrm{Y}$. The inverted fork transmits no association. If we condition on $\mathrm{L}$ (the collider), a non-causal association between $\mathrm{X}$ and $\mathrm{Y}$ is transmitted.

A path between $\mathrm{X}$ and $\mathrm{Y}$ is said to be $d$-separated if it contains a confounder or mediator that has been conditioned on, or a collider that has not been conditioned on (Pearl 1988). This implies that the path will not transmit any association; it is "blocked". In order for a statistical procedure to recover a causal association, it must be designed to block any non-causal paths. For example, in the DAG below, we wish to measure the causal association between $X$ and $Y$. There are, however, two non-causal paths that also connect $X$ to $Y$. The first is $X \leftarrow C \rightarrow Y$. This is a confounder path, and we close it by conditioning on $C$. The second non-causal path is $X \leftarrow A \rightarrow C \leftarrow B \rightarrow Y$. In this path the variable $C$ is not a confound but rather a collider. As a result, this path would normally be closed. But once we condition on $C$ to close the first path, it opens the second path. Therefore we must also condition on $A$ or $B$ to close this second path. Therefore a procedure which measures the association between $X$ and $Y$, stratified by $C$ and $B$ (or $A$; but using $B$ also increases precision (Pearl et al. 2016) and may thus be preferable) would measure the causal effect of $X$ on $Y$.

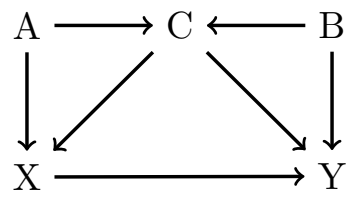




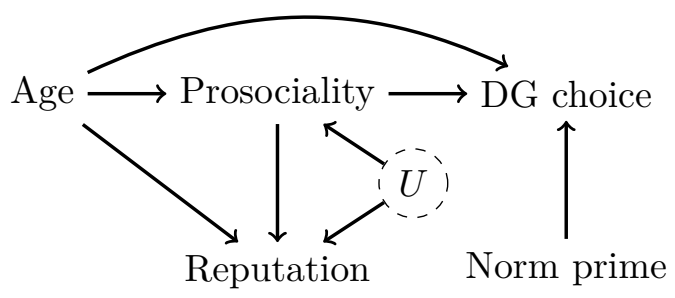

$(a)$

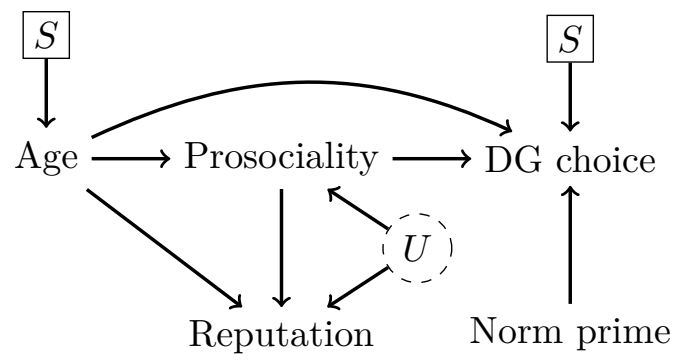

(b)

Figure 1. (a) A simple DAG capturing the following assumptions: Age has a direct causal effect on an individual's prosociality, their reputation within their community, as well as on the outcome of the Dictator game (DG). Prosociality and reputation share an unobserved common cause $U$. Prosociality in turn affects the individual's choice in the Dictator game, which is also affected by the randomized norm prime. (b) Selection diagram using selection nodes $S$ to represent the assumption that populations differ both in their age distribution and in the effect of norm primes on the choice in the dictator game.

Our DAG is of course incomplete and possibly wrong, in particular when it comes to the nodes that have not been experimentally manipulated. But an incomplete model is still an improvement over no model at all. In the absence of causal assumptions, whether in a DAG or otherwise, no analysis can be scientifically justified. Even an unrealistic DAG can help identify specific problems as well as implicit assumptions underlying more casually drawn causal inferences. Furthermore, such graphs make it easier to contrast the implications of different sets of assumptions which often lie at the heart of scientific disagreements. Throughout this article, we will use DAGs in this spirit - as a pragmatic tool to communicate assumptions and improve inference.

2.3. Selection Diagrams and Generalizability. DAGs can be extended to address generalizability through the use of selection diagrams (Pearl and Bareinboim 2014). When considering multiple populations, selection diagrams allow researchers to precisely define the local mechanisms by which populations are assumed to differ, as represented by "selection nodes". Selection nodes are not variables, but rather indicate which nodes have culture-specific distributions or causal relationships.

Returning to our previous example, in Figure 1b, we have added two selection nodes. The $S \rightarrow$ Age node may indicate that populations are characterized by different age distributions, the other $S$ node may indicate that the populations differ in the weight individuals give to norm primes when making decisions in the dictator game (recall that in a DAG, all variables that jointly affect another variable may interact). The absence of selection nodes in such graphs is of equal importance. It represents the assumption that certain mechanisms are the same across populations. For instance, the diagram in Fig. 1b implies that the development of prosociality with age does not vary among study populations. As shown below, it is this assumed invariance of certain mechanisms that makes generalizations possible.

Once we have a causal selection diagram, we can determine the scope for generalizability using logical rules. We can deduce when and how we can use data from one population to estimate a target quantity in another population, which is the central goal of the literature on transportability and data fusion (Pearl and Bareinboim 2014, Pearl 2015, Bareinboim and Pearl 2016, Cinelli and Pearl 2020). These logical rules can be compressed in most 
contexts to a set
(see section 3 ).

In cross-cultural settings, the research question often does not directly concern transport. Instead of transporting an estimate from one population to another, we instead have data sampled from multiple populations and want to make sense of the resulting numbers, learning more about whether, how, and why people differ from one another. However, such cross-cultural comparisons are still indirect exercises in transport, because in order to compare distributions or causal effects in different populations, we must calculate what those distributions or effects would be, if we changed the population.

2.4. Estimation: Multilevel regression with poststratification. After establishing 22 the logic of a generalization, one must actually compute it. For explicit generalization from sample to population and comparison across populations, we use multilevel regression with poststratification, a statistical technique that adjusts for differences between a sample population and a target population (Gelman and Little 1997, Gao et al. 2021, Wang et al. 2015). In a first step, the model uses partial pooling to obtain robust estimates for each "cell" (combination of attributes that we want to condition on; e.g., age/gender groups) taking into account information gained from other cells (Gelman and Hill 2006, McElreath 2020). For the data examples below, we use Gaussian processes to obtain estimates for each gender and age group while treating age as a continuous dimension; similar ages are expected to be similar in terms of their prosocial tendencies. In the second step (the "poststratification"), estimates for all cells are re-weighted using the relative frequencies of individuals per cell in the target population (see Appendix A for detailed explanation and model equations, and GitHub repository for Stan (Carpenter et al. 2017) code used to implement all analyses: https://github.com/DominikDeffner/Cross-Cultural-Generalizability).

Multilevel regression with poststratification enables us to learn from data and to project or "generalize" results to populations beyond the study sample in a principled way. Which population to use for poststratification depends on the theoretical estimand, the target of inference, and causal assumptions about the data generating process. Compared to more informal re-weighing procedures, multilevel regression with poststratification propagates uncertainty through all steps of analysis and is thus particularly suited for the small samples common in cross-cultural research.

Note that while the use of multilevel regression is not logically required - there are other estimation approaches - the use of poststratification is. The DAGs we will develop below mandate poststratification as a logical consequence of their structure. Informal re-weighting is only sometimes equivalent to this approach. In every case, the proper way to re-weight estimates is a consequence of causal assumptions.

\section{Applying the CAUSAl FRAMEWORK}

To illustrate our approach, we will use a large-scale cross-cultural project on societal diversity in prosocial behavior as an empirical case study (House et al. 2020). The researchers administered a binary-choice version of the dictator game as a measure of costly sharing to 255 adults and 833 children from eight populations spanning foragers, small-scale horticulturalists, and urban communities (see Fig. S3 in Appendix D for demographic composition of samples). Participants were asked to choose between a "self-maximizing" option where they would keep two rewards or a "prosocial" option where they would keep one reward and give one to an anonymous peer. Children from six societies were divided into three experimental conditions, where they viewed a short video with normative information before making their choices. These norm primes communicated which behavior was preferable ("Generous", "Both OK" or "Selfish"). We will use this rich data set as it exemplifies the 


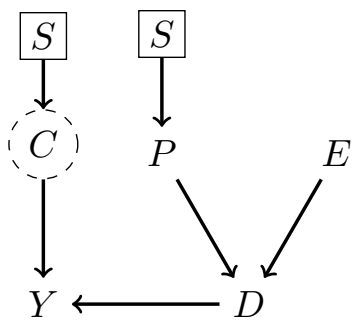

$(a)$

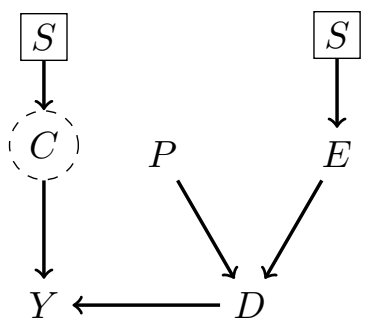

(b)

Figure 2. Different sources of demographic disparities among study samples. Prosociality $Y$ is caused by demography $D$ and unobserved cultural factors $C$. The sample demography $D$ is caused by population demography $\mathrm{P}$ and sampling procedures $E$. Selection nodes $S$ indicate mechanism by which populations differ. In addition to latent cultural factors, societies can differ in terms of (a) population demography or (b) sampling procedures.

state of the art in experimental cross-cultural research and excels with respect to research transparency.

3.1. Generalizing description: Cross-cultural comparisons and demographic standardization. A basic aim of cross-cultural research is to describe cultural variation. In the simplest case we might want to compare the prevalence of some institution or behavior across societies. This seemingly innocuous task of "pure description" may actually refer to a number of different research questions that call for different procedures. The example we provide is simplified and focuses on demography, but the point is not about demography. The same logic applies to all comparisons where populations differ in any known background factors.

3.1.1. Drawing out the causal assumptions. Samples from different sites often differ in terms of their demographic profiles (here: their age and gender distribution) and these demographic variables might in turn affect the distribution of the trait of interest.

How should researchers deal with these differences? The answer depends on the processes that generated the observed disparities. Demographic disparities among samples may result from (1) differences in the actual populations from which the samples are taken, or (2) sampling procedures that differ among sites. For example, if we observe that a sample from one site is on average younger than a sample from a second site, this may be because the underlying population is indeed younger. Alternatively, the difference could also result from a comparison of a relatively young convenience sample collected at one site with a full community sample at another site.

These scenarios are depicted in Figure 2. In this figure, an observed outcome $Y$ is influenced by both unobserved cultural factors $C$ and sample composition $D$. The sample composition is in turn influenced by the true demography $P$ and sampling procedures $E$ (for "experimenter").

If disparities arise from population differences (Fig. 2a), we can directly compare samples as long as our goal is to simply describe population differences in the focal trait $Y$, regardless of whether they arise from demography or from cultural factors. Adjustment is necessary, however, if we are are interested in different comparisons. For example, we may be interested in the counterfactual (i.e., hypothetical) distribution of the trait under comparable demographic profiles: If the two sites had comparable age and gender distributions, would we still observe differences in the trait of interest? This way, researchers could, for instance, 
290 isolate the influence of different cultural factors $C$ while holding constant demographic distributions. Note that such counterfactual comparisons might also correspond to a more substantive theoretical estimand, namely the distribution of a trait under a hypothetical intervention that moved individuals to another population (Lundberg et al. 2021).

If disparities among sites arise from different sampling procedures $E$ (Fig. 2b), even the purely descriptive question of observable population differences requires demographic adjustment, as sample demographics are systematically biased compared to the population of interest. For example, if the gender of the researcher influenced the gender of voluntary participants, then any differences between societies could be due to a mix of cultural, demographic, and sampling differences. In this case, even large samples do not accurately describe the target populations and we need to poststratify using information about the population from which samples are taken.

Another scenario, not illustrated in the figure, is when a sample is selected on the outcome variable $Y$ itself. For example, if prosocial individuals are more likely to cooperate with the researcher, this is selection on the outcome. In this case, there may be no solution to generalize from sample to population and therefore no way to compare populations. This is perhaps the starkest example of how description depends upon causal assumptions.

We will turn to real empirical data in the next subsection. However, knowing how to simulate data to validate an analytical strategy is also useful. In Appendix B, we walk through a complete simulated data example where we know the true generative process. We use multilevel regression with poststratification for the situation where populations differ in their demographic profile (Fig. S1, left column) and the complementary situation where demographic profiles of the populations are identical but genders are sampled unequally due to differences in local sampling procedures (Fig. S1, right column). In the first case, unadjusted empirical estimates accurately recover true population values, but poststratification can be used for counterfactual comparisons. In the second case, only poststratified estimates accurately recover true population values.

3.1.2. Empirical example. Let us turn to our empirical case study on prosociality across societies. Figure 3 shows a comparison between two actual populations included in House et al. (2020), Tanna island in Vanuatu (left) and Berlin in Germany (right). These societies have very different demographic profiles and sample compositions. Here, we are immediately confronted with a pragmatic concern: For many populations, no fine-grained demographic information is available. Therefore, we had to use the demography of all of Vanuatu instead of only Tanna. This highlights how collecting basic descriptive information about study populations is a crucial first step for any cross-cultural inference.

We divided data into 20 age categories spanning 5 years each and used Gaussian Process multilevel regression with poststratification (see Fig. S4 in Appendix D for gender- and agespecific model estimates). For Tanna, poststratification to either the demographic profile of Vanuatu or of Berlin leaves estimates unchanged (Figure 3, bottom row). This is because there was only a weak effect of age in this sample and the gender distribution was balanced. For the Berlin sample, on the other hand, adjusting for the demographic population profile of Berlin substantially increases the expected amount of prosociality. This results from the fact that older individuals in Berlin tended to be more prosocial in their choices and House et al. (2020) focused their data collection on children resulting in a much younger sample compared to the underlying population. Drawing the counterfactual comparison for Berlin individuals under the demographic profile of Vanuatu slightly increases the estimate.

How does this compare to the standard approach where researchers report raw age and gender specific estimates for each sample, thereby "controlling" for any differences? The 

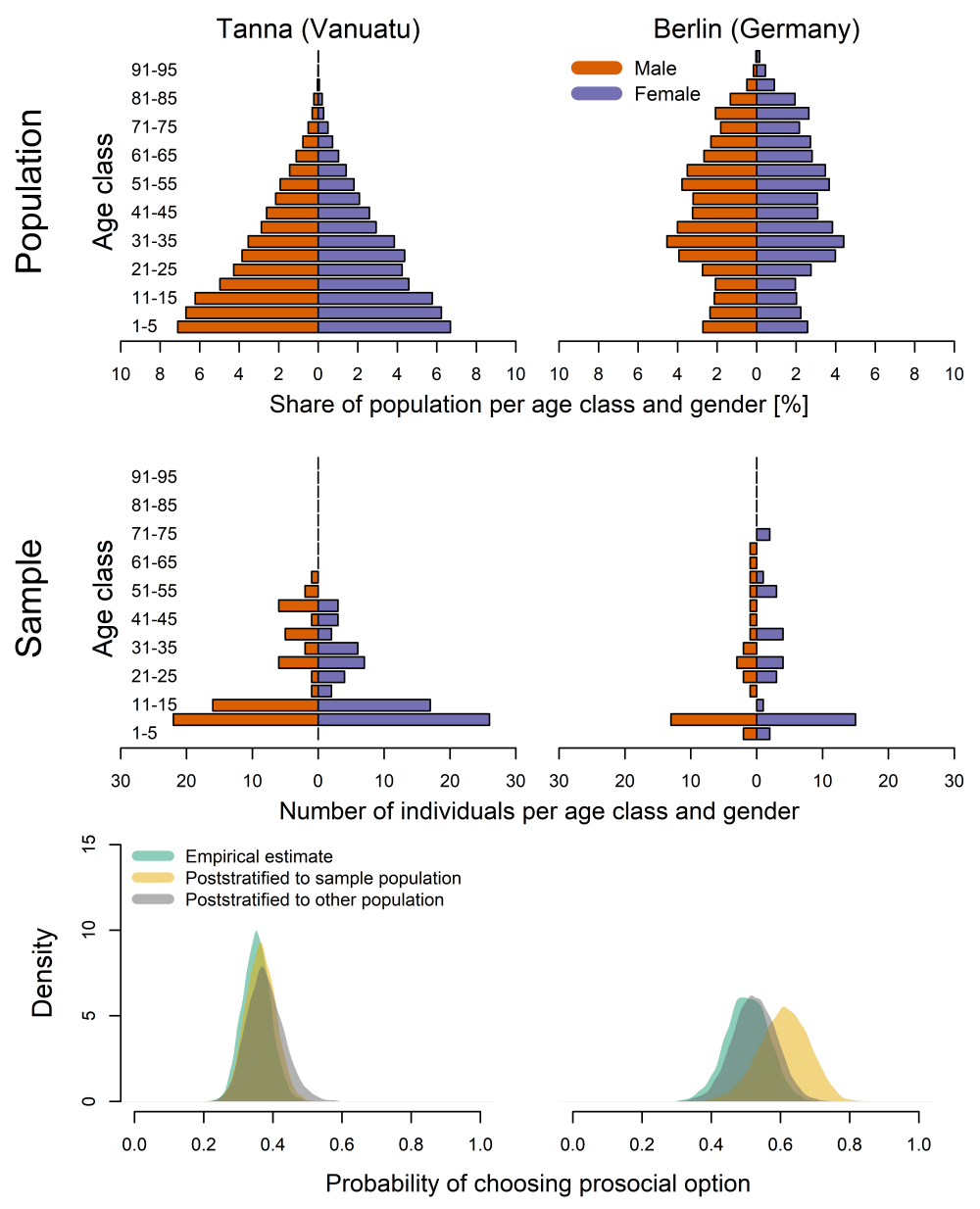

FiguRE 3. Data example for demographic standardization comparing prosociality among two populations, Tanna, Vanuatu (left) and Berlin, Germany (right). The top row shows demographic profiles of Vanuatu (UN Department of Economic and Social Affairs, World population prospects 2019) and Berlin (Mikrozensus 2020, Amt für Statistik Berlin-Brandenburg); the middle row shows demographic characteristics of study participants from both sites in House et al. (2020); the bottom row shows posterior distributions for probability to choose prosocial option from multilevel regression with poststratification analyses. Blue curves show empirical (unadjusted) estimates, yellow curves are poststratified to be representative of the population from which the sample was drawn, grey curves are poststratified to demographic profile of other population.

parameter estimates are necessary, but they are not enough. First, the claim that conditioning on age and gender controls for sample differences depends upon causal assumptions, as we explained in the previous sections. Second, the distribution of population differences depends not only upon the parameters but also upon the distributions of age and gender in each target population. A difference in parameters can look large but have little impact on population differences, both because the relevant age-gender categories may be too rare to make a large difference and because sizable differences on the parameter (e.g., logit) scale may result in minor differences on the outcome (e.g., probability) scale. Only by poststratifying to the outcome scale and to the relevant target population can behavioral differences be compared (Oganisian and Roy 2021, Rohrer and Arslan 2021).

While these examples have been simplified, they highlight the general concern. To accurately describe the prevalence of a trait and compare it across societies, we need to carefully define our theoretical estimand-consisting of unit-specific quantity and target 


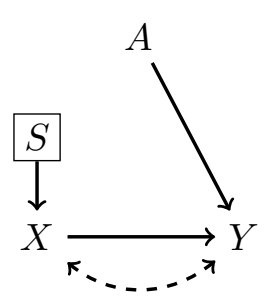

$(a)$

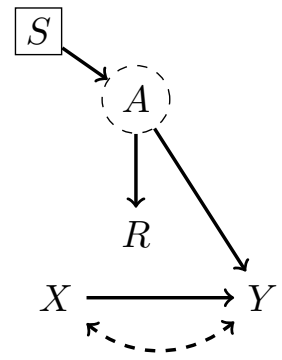

$(d)$

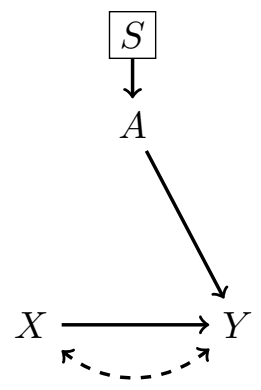

(b)

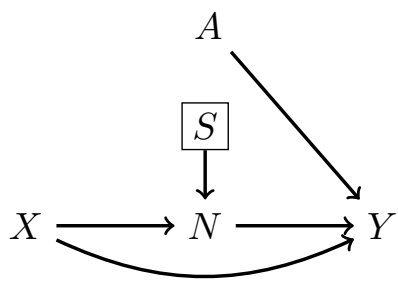

$(e)$

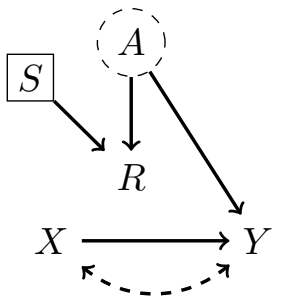

$(c)$

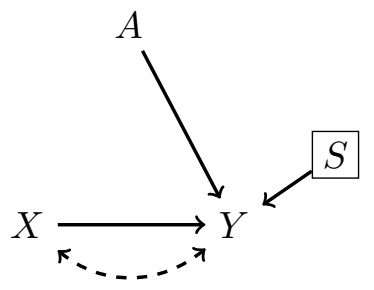

$(f)$

FIGURE 4. Scenarios for transportability of causal effects across populations: (a) Normative social information $X$, which is assumed to differ among populations, causes choice in dictator game $Y$; age $A$ modifies effect of $X$ on $Y$ but is invariant across populations; there are also unmeasured confounds between $X$ and $Y$ (indicated by dashed line); (b) Effect-modifier $A$ varies among populations; (c) Age itself is unobserved but we get to measure reported age $R$ as a proxy. It is assumed that the way people report their ages varies across societies, but the underlying age distribution is the same; (d) Ages are reported in the same way across populations but there are population differences in age distribution; (e) response in mediator variable, norm activation $N$, varies across societies; (f) populations vary in response of outcome variable $Y$ to treatment $X$. Note that scenarios (c), (d) and (e) are described in detail in appendix C.

population - and make assumptions about the processes that generate observed disparities in demography or any other potentially significant variable. Once a target population is set, refined statistical procedures, such as multilevel regression with poststratification, allow us to generalize observed outcomes to other populations conditional on causal assumptions. societies, but also the causal effect of an independent variable ("exposure", "treatment") on a dependent variable ("outcome"). In our example, we are interested in the causal effect of experimental norm primes on prosocial choices in the dictator game (House et al. 2020). Using the transportability framework from causal inference (Pearl 2015, Pearl and Bareinboim 2014), we show how causal thinking can be leveraged to generalize and compare causal effects across populations (see Appendix C for formal "S-admissibility"criterion).

Figure 4 shows selection diagrams for different scenarios varying in terms of scope and procedures for generalizations. They encode different sets of assumptions about the local mechanisms that cause populations to differ. We consider a situation where normative social information $X$ and age $A$ jointly cause choice in dictator game $Y$. Note these DAGs represent the "pre-treatment" situation, which means $X$ has not yet been experimentally set to a particular value. Once $X$ is manipulated through norm prime videos, all arrows entering $X$ (i.e., all "backdoor" paths) are deleted as the experimentalist is now the 

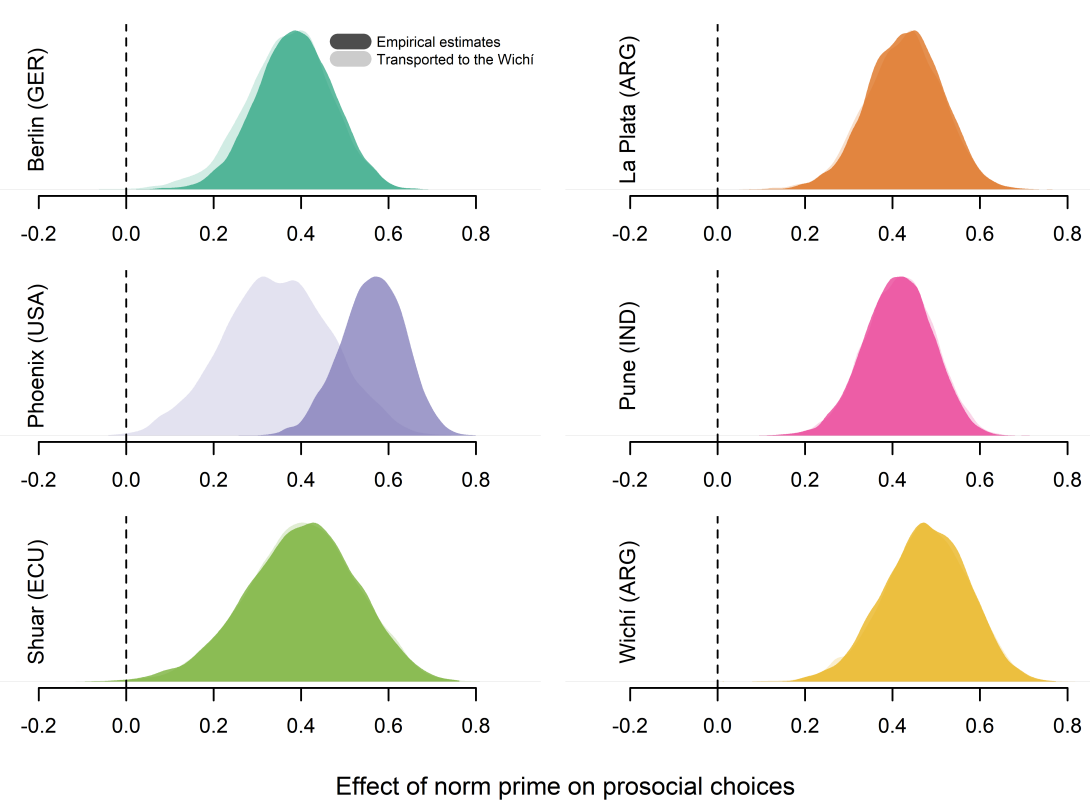

\begin{abstract}
Figure 5. Data example for transport of causal effects across societies. Empirical estimates (dark colors) and estimates transported to the Wichí in Argentina (transparent colors) for causal effect of norm primes ("Generous" vs. "Selfish") on prosocial choices in the dictator game in 6 different societies included in House et al. (2020). Estimates are calculated as the age-specific differences in the probability to choose the prosocial option in both conditions averaged over the age distribution in the target population.
\end{abstract}

370 sole cause of $X$. This allows us to estimate the causal effect from observed group differences. An experiment is necessary because we assume unobserved confounds-represented by dashed arrows - that influence both normative social information and prosocial choices (e.g., societies that strongly emphasize prosociality may be structured such that normative information is salient, but also encourage prosociality through other means).

375 3.2.1. Differences in independent/treatment variable. In Fig. 4a, populations differ in the distribution of normative social information $X$. This could mean, for instance, that in some societies individuals frequently encounter cultural narratives highlighting the importance of prosociality in their everyday life. As we have just seen, treatment randomization used in the experimental study cancels out such differences. As a consequence, the causal effect $380 \quad X \rightarrow Y$ is directly transportable or generalizable to other populations. In general, all selection nodes pointing into the independent variable (or other arrows that are removed in the $X$-manipulated graph) can be ignored (Pearl and Bareinboim 2014).

3.2.2. Differences in effect modifiers. The scenario depicted in Fig. $4 \mathrm{~b}$ is more interesting. Here, we assume population differences in age. As age modifies (or "moderates") the effect of normative information on choices (i.e., the effect of norm primes is assumed to be different for different ages) and the age distribution varies across populations, we cannot simply generalize the observed causal effect from one population to another. However, if age is assumed to affect the influence of norm primes in the same way across populations, we can estimate the age-specific effect of $X$ on $Y$ from experimental data and generalize by adjusting for the age distribution of the target population. 
The transport approach not only allows principled claims about the generalization of causal effects to new populations, it can also be employed to compare estimates from multiple populations from which experimental data are available. To determine if observed group differences in causal effects reflect "real" cultural differences, i.e., differences we cannot (yet) explain through other variables, or rather are due to sampling variation or differences in known effect modifiers, researchers need to make explicit assumptions about the causal processes that generate the data.

Figure 5 shows a data example for the transport of age-specific causal effects across populations (House et al. 2020). Because of experimental manipulation, the causal effect of norm primes $X$ on prosocial choices $Y$, our estimand, can be estimated from the difference in the probability to choose the prosocial option in both experimental conditions ("Generous" vs. "Selfish"). Dark colors show empirical estimates of this causal effect from 6 different societies included in House et al. (2020). Across all societies, posterior densities lay well above 0 . This means that individuals who watched the "Generous" prime video were substantially more likely to choose the prosocial option in the dictator game compared to individuals who watched the "Selfish" prime video, with the strongest effect being observed in the sample from Phoenix (USA).

To adjust for differences in the age distribution as a potential effect modifier, we estimate age-specific causal effects in each society and (as an example) adjust estimates to the demographic profile of the Wichí in Argentina. Transparent colors show such counterfactual estimates for the effect of norm primes in each society assuming it had the same demographic composition as the sample from the Wichí. While estimates remained largely unchanged for most societies, the effect for Phoenix becomes substantially smaller and more uncertain. This is because in Phoenix, age strongly modifies the effect of norm primes: younger children were more influenced by norm primes than older children. The Phoenix sample is, on average, almost three years younger than the Wichí sample, so estimates of the causal effect need to be adjusted to apply correctly to the Wichí demographic situation. Based on a comparison of naive empirical estimates, researchers might have wrongly concluded that norm primes have a particularly strong effect in Phoenix for some age-invariant cultural reason; transported estimates instead suggest that the larger effect is attributable to (potentially culturally determined) effect modification in combination with the younger sample. Adjustment for potential effect modifiers such as age, therefore, allows researchers to compare causal effects on an equal footing.

To aid understanding, most examples have been relatively straightforward, so some researchers might wonder what they gain from this causal approach compared to more informal ways to standardize and compare estimates across groups. Building up from those fundamental units, in Appendix C, we describe more complicated situations where implied generalizations and transport formulas could hardly be obtained by intuition alone.

In particular, Appendix $\mathrm{C}$ introduces scenarios where we do not observe the true effect modifier, biological age, but only some proxy such as reported age $R$, which is observed to vary across populations (Figs. 4c,d). As different scenarios will generate identical data distributions, the correct procedure will depend solely on causal assumptions. Appendix C further discusses situations where a mechanism mediating the effect of $X$ on $Y$ differs among societies (Fig. 4e) which requires a more sophisticated - yet algorithmically derivablegeneralization formula.

3.2.3. "Impossibility" of generalizations. Lastly, if a selection node is pointing directly into outcome variable $Y$ (Fig. 4f), no generalizations are possible because there is no immediate way to account for the source of disparity among populations (see Appendix $\mathrm{C}$ 


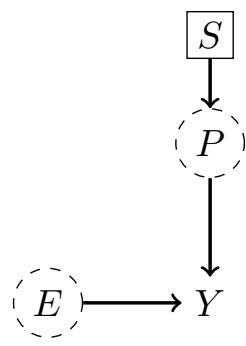

$(a)$

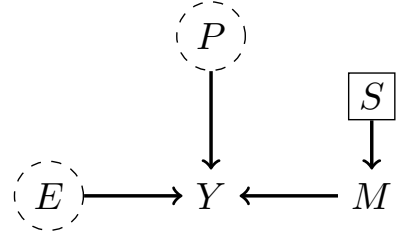

(b)

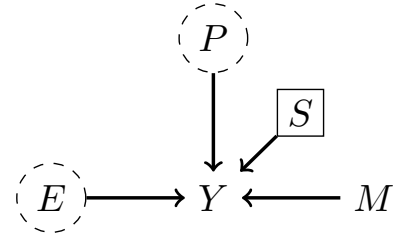

$(c)$

Figure 6. Causal representation of measurement (in-)equivalence across societies. (a) Choice in dictator game $Y$ is caused by latent psychological factor "Prosociality" $P$, which varies across populations, and unobserved sources of random error $E$. (b) Choice in dictator game $Y$ is also influenced by (population-specific) degree of market integration $M$. (c) The influence of prosociality $P$, error $E$ or market integration $M$ on observed choices differs among societies.

for "S-admissibility" criterion). This would be the case if unobserved population differences directly modify the effect (e.g., Oyserman and Lee 2008, found that individualismcollectivism primes do not function in comparable ways across societies), or if the form of age-modification varies between sites. However, even such "impossible" cases might allow generalizations and comparisons if researchers make additional assumptions; for example, if we have additional knowledge about the mechanisms causing the outcome variable, and analyzing effects of vitamin A supplementation on childhood mortality).

These examples demonstrate that the generalizability of experimental effects does not depend on the presence of population differences per se, but on the exact mechanisms by which populations differ. While some differences - especially those concerning the independent variable - are inconsequential for intended generalizations, differences concerning effect modifiers or mediators require statistical adjustment. Differences in the immediate mechanisms causing the outcome render generalizations difficult or even impossible. Such "real" cross-cultural differences may be the result of society-level factors directly influencing the trait of interest, and they present irreducible obstacles to generalization. Whether "real" cultural differences exist, or whether they must eventually be explained away by other mechanisms, is a topic beyond the scope of this paper.

3.3. Generalizing latent constructs: Measurement (in-)equivalence. In all examples so far, we have assumed that researchers can readily observe and measure the variables of interest. However, many (cross-cultural) psychologists are particularly interested in the comparison of latent constructs that are not directly observable. For example, researchers typically do not want to learn about dictator game choices per se, but about the underlying psychological constructs (e.g., "prosociality") which are assumed to generate the observed choices (see e.g., Pisor et al. 2020, Lesorogol 2007, Bond et al. 1982, Leung and Bond 1984, for potential impacts of cultural context on economic game choices). In this section, we will briefly demonstrate how causal selection diagrams can be used to represent common issues of measurement (in-)equivalence in cross-cultural studies; note that this is just a sketch, doing justice to this issue would require a whole paper.

Methodologists have long discussed whether and how data generated in cross-cultural research can be interpreted in terms of the presumed underlying processes and constructs. 470 The "equivalence and bias" framework, for instance, differentiates between construct equivalence, metric equivalence and scalar equivalence (e.g. Van de Vijver and Leung 2021, Van de 
Vijver and Tanzer 2004). Direct comparisons of measurements across societies are only justified if the underlying construct, measurement units and scale origin are equivalent across societies (i.e., full scalar equivalence).

475 But we can also approach the problem from a generative perspective. The measurement process can naturally be represented as a causal model of observed item or test scores (Borsboom et al. 2004, Bandalos 2018). It should be noted that there are alternative models where constructs are not seen as common causes of manifest variables, but as network structures (Borsboom et al. 2021) or organizing principles (Sijtsma 2006) that connect such variables; however, the implications of such models for generalizability are beyond the scope of this manuscript.

In Figure 6a, we assume that an individual's choice in the dictator game $Y$ is caused by a latent psychological factor $P$ (for "Prosociality") and unobserved sources of random "error" $E$. Measurement equivalence in this framework then requires that (1) only the distribution or the latent factor $P$ might vary across communities, (2) $P$ influences $Y$ in the same way everywhere, and (3) there are also no population differences in the unobserved error sources $E$. These conditions are fulfilled in Fig. 6a, so in this case we would be justified to compare game choices as indicators of latent "prosociality" across communities.

In Figure 6b, choices in the dictator game do not only reflect prosociality and random error, but also the degree of market integration $M$. People who engage more in market activities might be more likely to give a reward to an anonymous peer simply because they are more used to interact and trade with unknown others, not because they are more prosocial. In this case, choices in the dictator game are not equivalent measures of the latent factor in different societies, because they also include the influence of market integration which varies across societies. Nonetheless, following the logic on generalizing description (see section 3.1.), if we have data on market integration for each society, we can use poststratification to adjust for different levels of this variable and arrive at valid comparisons of prosociality and its causes across societies.

Lastly, Figure $6 \mathrm{c}$ shows a scenario where the selection node directly points into dictator game choice $Y$. This comprises situations of construct inequivalence where the latent construct itself is not comparable with respect to its influence on manifest behavior, but also cases where the influence of market integration or of unobserved error sources differs among societies. Mirroring the impossibility of transport with selection nodes pointing directly into outcome $Y$ (see Fig. 4f), in any such case, generalizations and comparisons about latent factors are unwarranted (unless additional assumptions are made). Because there is no way to statistically account for different sources of variation of observed choices $Y$, we cannot identify the unique influence of the latent state $P$ in equivalent ways across communities.

\section{USING THE CAUSAL FRAMEWORK FOR PRINCIPLED STUDY DESIGN}

A causal framework is not only useful for analysis, but also aids research design. In order to connect research designs to selection diagrams, we consider three stereotyped cases: a "maximally diverse" sampling strategy, a "proxy control" approach using phylogenetic distance or shared history, and a "regional comparative" approach that explicitly designs for local causal identification of the mechanisms by which populations differ. We explain each in turn.

515 A common approach in cross-cultural study design is to aim for "maximally diverse" populations. If effects can reliably be found across diverse societies, the reasoning goes, researchers are justified in assuming cross-cultural invariance or even universality; differences among samples are interpreted as evidence for either the influence of observed or unobserved cultural factors or methodological differences. By comparing geographically and culturally distant societies, this approach addresses "Galton's problem" which describes the pitfalls of 


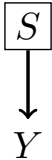

$(a)$

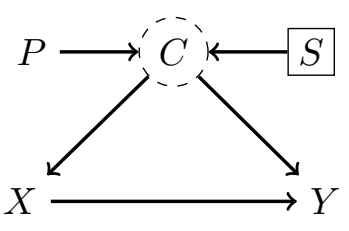

(b)

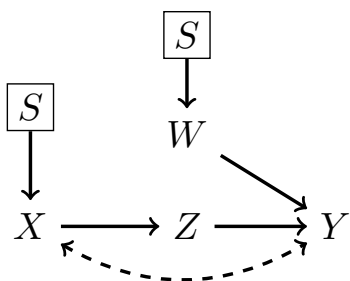

(c)

Figure 7. Different causal scenarios for study design: (a) Unobserved factors cause cross-cultural variation in outcome variable $Y$; (b) $X$ is a cause of $Y$, unobserved cultural variables $C$ that differ between populations influence both $X$ and $Y$; phylogenetic relationships $P$ influence $C$; (c) causal effect of $X$ is mediated by $Z$ and $W$ modifies the effect of $Z$ on $Y$. Dashed arrow represents unobserved common causes.

drawing inferences from cross-cultural data that are auto-correlated due to shared cultural and historical roots (Naroll 1965). This rationale guided the construction of the widelyused Standard Cross-Cultural Sample (Murdock and White 1969). Figure 7a encodes a scenario where researchers lack substantive theory on the factors causing a trait $Y$ that varies cross-culturally. Thus only a selection node is pointing into $Y$. As there is no way to separate sources of population differences from the trait itself, there are no theoretical grounds to predict how the trait might vary across populations. In such exploratory scenarios, it is advisable to sample many culturally distinct societies to approach a representative sample of the full range of variation (see section 3.1 for description of cultural variation). In general, when there is a selection node pointing directly into the outcome variable, researchers must incorporate relatively diverse populations as there are relevant but unknown variables causing population differences. However, potential dimensions of variation across settings, individuals, and societies are effectively infinite. They can never be sampled exhaustively, reflecting the classic problem of induction (Hume 1739, Sloman and Lagnado 2005). Additionally, while this approach reduces the chance that cross-cultural similarity is due to recent shared influences, it is not a general solution to causal inference, because any similarity between distant societies could still be due to unobserved variables.

A generalizable understanding of a given phenomenon, therefore, cannot be based only on the accumulation of data but requires the theory-driven testing of causal assumptions.

540 How even the most rudimentary causal theory helps increase generalizability can be seen in Fig. 7b, where researchers have identified an explanatory variable $X$. If researchers can find an identification strategy to estimate the causal effect $X \rightarrow Y$, they can leverage this causal knowledge to enhance generalizability following the transport approach outlined in section 3.2. The problem is that unobserved cultural variables $C$, which differ between

545 populations, influence both $X$ and $Y$ and thus confound the causal effect. One approach is to try to model the covariation among populations that arises from such unobserved confounds. Variables like geographic, linguistic or cultural distance $P$ can be used as proxies to control for unmeasured common causes of similarity. The notion is that populations closer in space or cultural history share more unmeasured common causes. This can permit causal investigation of, for example, ecological and demographic factors in otherwise opportunistic collections of societies. Various cultural and linguistic phylogenetic methods try to implement this strategy (see section 14.5 of McElreath 2020, for detailed examples). This approach makes strong causal assumptions about the nature of confounding and our ability to measure shared history. However, strong assumptions are always necessary in observa555 tional settings. What is important is that the assumptions are transparent and logically connected to data analysis. 
Finally, Figure 7c shows a scenario where researchers have developed more mechanistic theory including additional variables lying on the causal paths between selection nodes and outcome; this provides more principled expectations about the mechanisms generating population disparities. Specifically, there is an intermediate variable $Z$ mediating the effect of $X$ on $Y$ and another variable $W$ that modifies the effect of $Z$ on $Y$. Assuming this DAG, there is no selection node pointing into $Y$ anymore, and thus researchers can explain all population differences in the focal trait based on the joint causal effects of other variables. A research design that attempts to address causation directly is the "regional comparative" approach. In this approach, researchers explicitly target closely related societies that only differ in key variables of interest (Johnson 1991, Boas 1896). By holding other factors constant, such "quasi-experimental" comparisons among regional (sub-)populations allow researchers to isolate the effect of a variable of interest and facilitate causal inference. This strategy is similar to difference-in-difference (Lechner 2011) and regression discontinuity designs (Imbens and Lemieux 2008, Lee and Lemieux 2010). A classic example of the approach is the Culture and Ecology in East Africa Project that compared samples from four different ethnic groups, each of which comprised neighboring pastoralist and horticulturalist communities in different but adjacent ecologies (Goldschmidt 1965, Edgerton 1971). While differences among ethnic groups are hard to interpret, differences between neighboring communities within each ethnic group are arguably due to local ecological and economic differences (see Mattison et al. 2016, Glowacki and Molleman 2017, for more recent examples).

Summarizing, because of the problem of induction, generalizability can never be determined through the accumulation of cross-cultural data alone and requires the development of formal theory to accompany and guide cross-cultural data collection (Muthukrishna and Henrich 2019). The maximally informative research design depends on the state of mechanistic understanding of the phenomenon of interest. By explicitly stating and refining the causal assumptions underlying population differences, researchers can target maximally informative cross-cultural comparisons, generating results that are not only grounded in theory but also generalizable beyond the immediate study samples.

More diverse samples are urgently needed, but they bring forth new conceptual challenges for description, generalization and comparison. The accumulation of large cross-cultural datasets in combination with lists of threats to validity allows only limited progress. What is needed in addition is a structural causal modeling framework. An explicit causal framework empowers researchers by providing a way to plan cross-cultural comparisons, to implement and justify analyses, and to determine which interpretations are warranted under which sets of assumptions. It also provides a powerful way to critically and fairly evaluate the studies of others and to formally represent sources of disagreement. An effective critique should aim for the same causal clarity as an effective study. When an original study lacks causal clarity, an effective critique may identify which causal model is implied by the analysis, and subsequently assess the plausibility of specific elements.

Researchers in various fields already apply methods that address some of the concerns we discussed above. For example, political scientists and sociologists apply demographic standardization (e.g., Kitagawa decomposition) to estimate effects of interventions for counterfactual populations (Kitagawa 1955, Preston et al. 2000, Acharya et al. 2016, Ciocca Eller and DiPrete 2018, Mize 2016, Storer et al. 2020, Ross et al. 2021). Anthropologists calculate age-corrected values to standardize across populations (Borgerhoff Mulder et al. 2009, Mattison et al. 2016, Jaeggi et al. 2021, Rowan et al. 2021). Economists calculate average treatment effects (ATEs) and marginal effects that can take into account effect modification by demographic variables (Morgan and Winship 2015, Athey and Imbens 2016, Greene 2000, 
Asteriou and Hall 2015); and the Heckman correction is applied to account for non-random sample selection (Heckman 1976, 1979, Puhani 2000). And even simple regression controls can account for population differences in background factors in some limited situations.

The framework we champion - poststratification and transport based on causal graphsgoes beyond these partial solutions. It is explicit about the target of inference and the assumptions that justify the analysis; it logically derives statistical procedures from a generative causal model. Therefore, it is more general and unifies a large number of inferential concerns (e.g., confounding, selection bias, standardization, generalization) within a common framework. Likewise, the estimation strategy that we propose - multilevel regression with poststratification - is very flexible. It allows to project estimates to arbitrary target populations and can account for any number of variables and functional relationships between them. In contrast, simply including age and sex as covariates in multiple regression assumes that all relationships are linear and estimates population differences holding covariates constant at an arbitrary level. Under the right circumstances, this standard approach might tell us something about differences between observed samples, but does not enable us to generalize findings to the sample populations (in case of sampling differences) and other populations in a reasoned way.

It is quite obvious that all the scenarios we presented were oversimplified. An explicit causal inference framework makes it (at times painfully) transparent how strong the assumptions are that we need to arrive at substantive conclusions, and how little we collectively know about many real-world phenomena. But this is no reason to embrace the status quo that often avoids causal language (Grosz et al. 2020) - assumptions do not disappear just because we ignore them. Cross-cultural research is daunting, and strong conclusions require strong methods for data collection, its description, and its analysis. A structural causal framework encourages researchers to explicitly spell out their assumptions, removing verbal ambiguity and facilitating communication; and it calls for a cumulative approach to science as one study's findings become the scaffolding assumptions of the next.

Data Availability. This manuscript does not contain any new empirical data. Analysis, simulation and plotting code necessary to reproduce all results and figures in the manuscript can be found on GitHub: https://github.com/DominikDeffner/Cross-CulturalGeneralizability.

Acknowledgements. We thank members of the Department for Human Behavior, Ecology and Culture and the Department of Comparative Cultural Psychology at the Max Planck Institute for Evolutionary Anthropology in Leipzig for constructive discussions and criticisms which helped improving this paper.

Authors' Contributions. DD and RM conceived the project. DD wrote the simulations and performed the analyses for the data examples. DD, JMR and RM wrote the manuscript.

Competing Interests. The authors declare no competing interests. This work has been funded by the Max Planck Society.

\section{REFERENCES}

Acharya, A., Blackwell, M., and Sen, M. (2016). Explaining causal findings without bias: Detecting and assessing direct effects. American Political Science Review, 110(3):512-529.

Apicella, C., Norenzayan, A., and Henrich, J. (2020). Beyond weird: A review of the last decade and a look ahead to the global laboratory of the future. Evolution and Human Behavior, 41(5):319-329.

Asteriou, D. and Hall, S. G. (2015). Applied econometrics. Macmillan International Higher Education.

Athey, S. and Imbens, G. (2016). Recursive partitioning for heterogeneous causal effects. Proceedings of the National Academy of Sciences, 113(27):7353-7360. 
Awad, E., Dsouza, S., Kim, R., Schulz, J., Henrich, J., Shariff, A., Bonnefon, J.-F., and Rahwan, I. (2018). The moral machine experiment. Nature, 563(7729):59-64.

Awad, E., Dsouza, S., Shariff, A., Rahwan, I., and Bonnefon, J.-F. (2020). Universals and variations in moral decisions made in 42 countries by 70,000 participants. Proceedings of the National Academy of Sciences, 117(5):2332-2337.

Bandalos, D. L. (2018). Measurement theory and applications for the social sciences. Guilford Publications.

Bareinboim, E. and Pearl, J. (2016). Causal inference and the data-fusion problem. Proceedings of the National Academy of Sciences, 113(27):7345-7352.

Barrett, H. C. (2020). Deciding what to observe: Thoughts for a post-weird generation. Evolution and Human Behavior, 41(5):445-453.

Berkowitz, L. and Donnerstein, E. (1982). External validity is more than skin deep: Some answers to criticisms of laboratory experiments. American psychologist, 37(3):245.

Boas, F. (1896). The limitations of the comparative method of anthropology. Science, 4(103):901908.

Bond, M. H., Leung, K., and Wan, K. C. (1982). How does cultural collectivism operate? the impact of task and maintenance contributions on reward distribution. Journal of Cross-Cultural Psychology, 13(2):186-200.

Borgerhoff Mulder, M., Bowles, S., Hertz, T., Bell, A., Beise, J., Clark, G., Fazzio, I., Gurven, M., Hill, K., Hooper, P. L., et al. (2009). Intergenerational wealth transmission and the dynamics of inequality in small-scale societies. Science, 326(5953):682-688.

Borsboom, D., Deserno, M. K., Rhemtulla, M., Epskamp, S., Fried, E. I., McNally, R. J., Robinaugh, D. J., Perugini, M., Dalege, J., Costantini, G., et al. (2021). Network analysis of multivariate data in psychological science. Nature Reviews Methods Primers, 1(1):1-18.

Borsboom, D., Mellenbergh, G. J., and Van Heerden, J. (2004). The concept of validity. Psychological review, 111(4):1061.

Broesch, T., Crittenden, A. N., Beheim, B. A., Blackwell, A. D., Bunce, J. A., Colleran, H., Hagel, K., Kline, M., McElreath, R., Nelson, R. G., et al. (2020). Navigating cross-cultural research: methodological and ethical considerations. Proceedings of the Royal Society B, 287(1935):20201245.

Calder, B. J., Phillips, L. W., and Tybout, A. M. (1983). Beyond external validity. Journal of Consumer Research, 10(1):112-114.

Campbell, D. T. (1957). Factors relevant to the validity of experiments in social settings. Psychological bulletin, 54(4):297.

Carpenter, B., Gelman, A., Hoffman, M. D., Lee, D., Goodrich, B., Betancourt, M., Brubaker, M., Guo, J., Li, P., and Riddell, A. (2017). Stan: A probabilistic programming language. Journal of statistical software, 76(1).

Cinelli, C., Forney, A., and Pearl, J. (2020). A crash course in good and bad controls. Available at SSRN.

Cinelli, C. and Pearl, J. (2020). Generalizing experimental results by leveraging knowledge of mechanisms. European Journal of Epidemiology, pages 1-16.

Ciocca Eller, C. and DiPrete, T. A. (2018). The paradox of persistence: Explaining the black-white gap in bachelor's degree completion. American Sociological Review, 83(6):1171-1214.

Clancy, K. B. and Davis, J. L. (2019). Soylent is people, and weird is white: Biological anthropology, whiteness, and the limits of the weird. Annual Review of Anthropology, 48:169-186.

Cock, T. and Campbell, D. (1976). The design and conduct of quasi-experiments and true experiments in field setting. Handbook of Industrial and Organizational Psychology, Chicago: Rand McNally, pages 223-326.

Curtin, C. M., Barrett, H. C., Bolyanatz, A., Crittenden, A. N., Fessler, D. M., Fitzpatrick, S., Gurven, M., Kanovsky, M., Kushnick, G., Laurence, S., et al. (2020). Kinship intensity and the use of mental states in moral judgment across societies. Evolution and Human Behavior, 41(5):415-429.

Edgerton, R. B. (1971). The individual in cultural adaptation: A study of four East African peoples. University of California Press. 
Elwert, F. (2013). Graphical causal models. In Morgan, S. L., editor, Handbook of Causal Analysis for Social Research, pages 245-273. Springer Netherlands, Dordrecht.

Falk, A., Becker, A., Dohmen, T., Enke, B., Huffman, D., and Sunde, U. (2018). Global evidence on economic preferences. The Quarterly Journal of Economics, 133(4):1645-1692.

Farrell, S. and Lewandowsky, S. (2018). Computational modeling of cognition and behavior. Cambridge University Press.

Gächter, S. and Schulz, J. F. (2016). Intrinsic honesty and the prevalence of rule violations across societies. Nature, 531(7595):496-499.

Gao, Y., Kennedy, L., Simpson, D., Gelman, A., et al. (2021). Improving multilevel regression and poststratification with structured priors. Bayesian Analysis.

Gelman, A. and Hill, J. (2006). Data analysis using regression and multilevel/hierarchical models. Cambridge university press.

Gelman, A. and Little, T. C. (1997). Poststratification into many categories using hierarchical logistic regression.

Ghai, S. (2021). It's time to reimagine sample diversity and retire the weird dichotomy. Nature Human Behaviour, pages 1-2.

Glowacki, L. and Molleman, L. (2017). Subsistence styles shape human social learning strategies. Nature human behaviour, 1(5):1-5.

Goldschmidt, W. (1965). Theory and strategy in the study of cultural adaptability. American Anthropologist, 67(2):402-408.

Greene, W. H. (2000). Econometric analysis 4th edition. International edition, New Jersey: Prentice Hall, pages 201-215.

Grosz, M. P., Rohrer, J. M., and Thoemmes, F. (2020). The taboo against explicit causal inference in nonexperimental psychology. Perspect. Psychol. Sci., 15(5):1243-1255.

Heckman, J. J. (1976). The common structure of statistical models of truncation, sample selection and limited dependent variables and a simple estimator for such models. In Annals of economic and social measurement, volume 5, number 4, pages 475-492. NBER.

Heckman, J. J. (1979). Sample selection bias as a specification error. Econometrica: Journal of the econometric society, pages 153-161.

Henrich, J. (2020). The WEIRDest people in the world: How the West became psychologically peculiar and particularly prosperous. Farrar, Straus and Giroux.

Henrich, J., Boyd, R., Bowles, S., Camerer, C., Fehr, E., Gintis, H., and McElreath, R. (2001). In search of homo economicus: behavioral experiments in 15 small-scale societies. American Economic Review, 91(2):73-78.

Henrich, J., Heine, S. J., and Norenzayan, A. (2010). Beyond weird: Towards a broad-based behavioral science. Behavioral and Brain Sciences, 33(2-3):111.

House, B. R., Kanngiesser, P., Barrett, H. C., Broesch, T., Cebioglu, S., Crittenden, A. N., Erut, A., Lew-Levy, S., Sebastian-Enesco, C., Smith, A. M., et al. (2020). Universal norm psychology leads to societal diversity in prosocial behaviour and development. Nature Human Behaviour, $4(1): 36-44$.

Hume, D. (2003/1739). A treatise of human nature. Dover Publications, INC.

Imbens, G. W. and Lemieux, T. (2008). Regression discontinuity designs: A guide to practice. Journal of econometrics, 142(2):615-635.

Jaeggi, A. V., Blackwell, A. D., von Rueden, C., Trumble, B. C., Stieglitz, J., Garcia, A. R., Kraft, T. S., Beheim, B. A., Hooper, P. L., Kaplan, H., et al. (2021). Do wealth and inequality associate with health in a small-scale subsistence society? Elife, 10:e59437.

Johnson, A. (1991). Regional comparative field research. Behavior Science Research, 25(1-4):3-22.

Jones, B. C., DeBruine, L. M., Flake, J. K., Liuzza, M. T., Antfolk, J., Arinze, N. C., Ndukaihe, I. L., Bloxsom, N. G., Lewis, S. C., Foroni, F., et al. (2021). To which world regions does the valence-dominance model of social perception apply? Nature human behaviour, pages 1-9.

Kitagawa, E. M. (1955). Components of a difference between two rates. Journal of the american statistical association, 50(272):1168-1194.

Lechner, M. (2011). The estimation of causal effects by difference-in-difference methods. Now Hanover, MA. 
Lee, D. S. and Lemieux, T. (2010). Regression discontinuity designs in economics. Journal of economic literature, 48(2):281-355.

Lesorogol, C. K. (2007). Bringing norms in: the role of context in experimental dictator games. Current anthropology, 48(6):920-926.

Leung, K. and Bond, M. H. (1984). The impact of cultural collectivism on reward allocation. Journal of Personality and Social psychology, 47(4):793.

Lundberg, I., Johnson, R., and Stewart, B. M. (2021). What is your estimand? defining the target quantity connects statistical evidence to theory. American Sociological Review, 86(3):532-565.

Masuda, T. and Nisbett, R. E. (2001). Attending holistically versus analytically: comparing the context sensitivity of japanese and americans. Journal of personality and social psychology, 81(5):922.

Matthay, E. C. and Glymour, M. M. (2020). A graphical catalog of threats to validity: linking social science with epidemiology. Epidemiology (Cambridge, Mass.), 31(3):376.

Mattison, S. M., Beheim, B., Chak, B., and Buston, P. (2016). Offspring sex preferences among patrilineal and matrilineal mosuo in southwest china revealed by differences in parity progression. Royal Society Open Science, 3(9):160526.

McElreath, R. (2020). Statistical rethinking: A Bayesian course with examples in $R$ and Stan. CRC press.

Mehr, S. A., Singh, M., Knox, D., Ketter, D. M., Pickens-Jones, D., Atwood, S., Lucas, C., Jacoby, N., Egner, A. A., Hopkins, E. J., et al. (2019). Universality and diversity in human song. Science, $366(6468)$.

Mize, T. D. (2016). Sexual orientation in the labor market. American Sociological Review, 81(6):1132-1160.

Morgan, S. L. and Winship, C. (2015). Counterfactuals and causal inference. Cambridge University Press.

Murdock, G. P. and White, D. R. (1969). Standard cross-cultural sample. Ethnology, 8(4):329-369.

Muthukrishna, M., Bell, A. V., Henrich, J., Curtin, C. M., Gedranovich, A., McInerney, J., and Thue, B. (2020). Beyond western, educated, industrial, rich, and democratic (weird) psychology: Measuring and mapping scales of cultural and psychological distance. Psychological Science, 31(6):678-701.

Muthukrishna, M. and Henrich, J. (2019). A problem in theory. Nature Human Behaviour, 3(3):221229.

Naroll, R. (1965). Galton's problem: The logic of cross-cultural analysis. Social Research, pages $428-451$.

Nisbett, R. E. and Miyamoto, Y. (2005). The influence of culture: holistic versus analytic perception. Trends in cognitive sciences, 9(10):467-473.

Norenzayan, A. and Heine, S. J. (2005). Psychological universals: What are they and how can we know? Psychological bulletin, 131(5):763.

Oganisian, A. and Roy, J. A. (2021). A practical introduction to bayesian estimation of causal effects: Parametric and nonparametric approaches. Statistics in Medicine, 40(2):518-551.

Oyserman, D. and Lee, S. W. (2008). Does culture influence what and how we think? effects of priming individualism and collectivism. Psychological bulletin, 134(2):311-342.

Pearl, J. (1988). Probabilistic Reasoning in Intelligent Systems: Networks of Plausible Inference. Morgan Kaufmann Publishers Inc.

Pearl, J. (2015). Generalizing experimental findings. Journal of Causal Inference, 3(2):259-266.

Pearl, J. (2018). Theoretical impediments to machine learning with seven sparks from the causal revolution. arXiv preprint arXiv:1801.04016.

Pearl, J. and Bareinboim, E. (2014). External validity: From do-calculus to transportability across populations. Statistical Science, pages 579-595.

Pearl, J., Glymour, M., and Jewell, N. P. (2016). Causal inference in statistics: A primer. John Wiley \& Sons.

Pearl, J. and Mackenzie, D. (2018). The book of why: the new science of cause and effect. Basic books.

Pisor, A. C., Gervais, M. M., Purzycki, B. G., and Ross, C. T. (2020). Preferences and constraints: the value of economic games for studying human behaviour. Royal Society open science, 
7(6):192090.

Pollet, T. V., Tybur, J. M., Frankenhuis, W. E., and Rickard, I. J. (2014). What can cross-cultural correlations teach us about human nature? Human Nature, 25(3):410-429.

Preston, S., Heuveline, P., and Guillot, M. (2000). Demography: Measuring and Modeling Population Processes. Wiley-Blackwell.

Puhani, P. (2000). The heckman correction for sample selection and its critique. Journal of economic surveys, $14(1): 53-68$.

Rohrer, J. M. (2018). Thinking clearly about correlations and causation: Graphical causal models for observational data. Advances in Methods and Practices in Psychological Science, 1(1):27-42.

Rohrer, J. M. and Arslan, R. C. (2021). Precise answers to vague questions: Issues with interactions. Advances in Methods and Practices in Psychological Science, 4(2):25152459211007368.

Ross, C. T., Winterhalder, B., and McElreath, R. (2021). Racial disparities in police use of deadly force against unarmed individuals persist after appropriately benchmarking shooting data on violent crime rates. Social Psychological and Personality Science, 12(3):323-332.

Rowan, C. J., Eskander, M. A., Seabright, E., Rodriguez, D. E., Linares, E. C., Gutierrez, R. Q., Adrian, J. C., Cummings, D., Beheim, B., Tolstrup, K., et al. (2021). Very low prevalence and incidence of atrial fibrillation among bolivian forager-farmers. Annals of global health, 87(1).

Rozin, P. (2001). Social psychology and science: Some lessons from solomon asch. Pers. Soc. Psychol. Rev., 5(1):2-14.

Shadish, W. R., Cook, T. D., Campbell, D. T., et al. (2002). Experimental and quasi-experimental designs for generalized causal inference/William R. Shedish, Thomas D. Cook, Donald T. Campbell. Boston: Houghton Mifflin,.

Sijtsma, K. (2006). Psychometrics in psychological research: Role model or partner in science? Psychometrika, 71(3):451-455.

Simons, D. J., Shoda, Y., and Lindsay, D. S. (2017). Constraints on generality (cog): A proposed addition to all empirical papers. Perspectives on Psychological Science, 12(6):1123-1128.

Sloman, S. A. and Lagnado, D. (2005). The problem of induction. The Cambridge handbook of thinking and reasoning, pages $95-116$.

Smaldino, P. E., Lukaszewski, A., von Rueden, C., and Gurven, M. (2019). Niche diversity can explain cross-cultural differences in personality structure. Nature Human Behaviour, 3(12):12761283.

Storer, A., Schneider, D., and Harknett, K. (2020). What explains racial/ethnic inequality in job quality in the service sector? American Sociological Review, 85(4):537-572.

Tiokhin, L., Hackman, J., Munira, S., Jesmin, K., and Hruschka, D. (2019). Generalizability is not optional: insights from a cross-cultural study of social discounting. Royal Society Open Science, 6(2):181386.

Urassa, M., Lawson, D. W., Wamoyi, J., Gurmu, E., Gibson, M. A., Madhivanan, P., and Placek, C. (2021). Cross-cultural research must prioritize equitable collaboration. Nature Human Behaviour, pages $1-4$.

Van de Vijver, F. and Tanzer, N. K. (2004). Bias and equivalence in cross-cultural assessment: An overview. European Review of Applied Psychology, 54(2):119-135.

Van de Vijver, F. J. and Leung, K. (2021). Methods and data analysis for cross-cultural research, volume 116. Cambridge University Press.

Wang, W., Rothschild, D., Goel, S., and Gelman, A. (2015). Forecasting elections with nonrepresentative polls. International Journal of Forecasting, 31(3):980-991.

Winer, R. S. (1999). Experimentation in the 21st century: The importance of external validity. Journal of the Academy of marketing Science, 27(3):349.

Woodward, J. (2005). Making things happen: A theory of causal explanation. Oxford university press.

Yarkoni, T. (2020). The generalizability crisis. Behavioral and Brain Sciences, pages 1-37. 


\title{
Supplementary material for
}

\section{"A Causal Framework for Cross-Cultural Generalizability"}

\section{Dominik Deffner ${ }^{1,2,3^{*}}$, Julia M. Rohrer ${ }^{4} \&$ Richard McElreath $^{1}$}

\author{
${ }^{1}$ Department of Human Behavior, Ecology and Culture, Max Planck Institute for Evolutionary An- \\ thropology, Leipzig, Germany \\ ${ }^{2}$ Science of Intelligence Excellence Cluster, Technical University Berlin, Berlin, Germany \\ ${ }^{3}$ Center for Adaptive Rationality, Max Planck Institute for Human Development, Berlin, Germany \\ ${ }^{4}$ Department of Psychology, Leipzig University, Leipzig, Germany \\ *Corresponding author: deffner@mpib-berlin.mpg.de
}

\section{Overview}

Appendix A explains statistical details of multilevel regression with poststratification (MRP) and transport analyses using Gaussian processes.

Appendix B shows simulated data example for demographic standardization.

Appendix C explains "S-admissibility" and transport procedures for proxy effect modifiers and differences in mechanism/mediator variables.

Appendix D contains supplementary figures.

\section{A. Statistical analyses}

Multilevel regression with poststratification

As suggested by the name, multilevel regression with poststratification (MRP) consists of two basic components. First, multilevel models - also known as hierarchical, random effects, varying effects or mixed effects models - use partial pooling to obtain robust estimates for each "cell" (combination of attributes; e.g., age/gender groups) while considering information gained from other cells (Gelman and Hill, 2006; McElreath, 2020). This is achieved by estimating single parameters for each cell while simultaneously estimating so-called hyperparameters for the joint distribution of those parameters among cells. Here, we use Gaussian processes to obtain robust estimates for each gender and age group while treating age as a continuous dimension where similar ages are expected to be similar in terms of their prosocial tendencies. Gaussian processes extend the multilevel approach to continuous categories such as age and estimate a unique parameter value for each level, while still regarding age as a continuous dimension in which similar levels are characterized by more similar behavior (McElreath, 2020). Specifically, the probability to choose the prosocial option for individual $i$ with gender $j$ and age $k$ is composed of the average for each gender and an age-specific offset:

$$
\operatorname{Pr}\left(y_{i}=1\right)=\operatorname{logit}^{-1}\left(\alpha_{j[i]}+d_{j_{[i]}, k_{[i]}}\right) .
$$

We define a multivariate Gaussian prior (hence the name "Gaussian process") for the age-specific offsets $d_{j, k}$ separately for two genders: 


$$
\left(\begin{array}{c}
d_{j, 1} \\
d_{j, 2} \\
\cdots \\
d_{j, \text { age } \max }
\end{array}\right) \sim N\left[\left(\begin{array}{c}
0 \\
0 \\
\ldots \\
0
\end{array}\right), \quad K\right] .
$$

The vector of means is all zeros, so the average gender effect remains unchanged, and $K$ is the covariance matrix among all age levels. We estimate the parameters of a function that expresses how the covariance between different ages is expected to change as the distance increases:

$$
K_{x, y}=\eta_{j}^{2} \exp \left(-\rho_{j}^{2} D_{x, y}^{2}\right)+\delta_{x, y} \sigma_{j}^{2} .
$$

The covariance between any age pair $x$ and $y, K_{x, y}$, equals the maximum covariance $\eta_{j}^{2}$ which is reduced at rate $\rho_{j}^{2}$ by the squared distance in age between $x$ and $y, D_{x, y}^{2}$. There is an additional covariance parameter $\sigma_{j}^{2}$ that gets "turned on" by $\delta_{x, y}$ when $x=y$; it expresses the additional covariance for observations of participants with the same age. These control parameters have a subscript $j$ because they could vary by gender.

The output of this first part of the model is an estimate of the average response for each cross classification $l$ of gender and age, $\pi_{l}$. In principle, this approach can be extended to multiple demographic or other variables which then results in a high number of unique combinations. In the second step (the "poststratification"), estimates for all cells $\pi_{l}$ are re-weighted by the frequency of individuals per cell in the target population $N_{l}$ resulting in the poststratified (i.e. adjusted) estimate $\theta$ :

$$
\theta=\frac{\sum_{l} N_{l} \pi_{l}}{\sum_{l} N_{l}} .
$$

\section{Transport}

The general procedure for transporting causal effects across populations is very similar. In the first step, we use a binomial model to predict the effect of norm primes $P$ on prosocial choices $y$. This effect is composed of the average effect $\bar{\beta}_{\text {prime }}$ and an age-specific offset $d_{k_{[i]}}$ :

$$
\operatorname{Pr}\left(y_{i}=1\right)=\operatorname{logit}^{-1}\left[\alpha+\left(\bar{\beta}_{\text {prime }}+d_{k_{[i]}}\right) P_{[i]}\right] .
$$

As before, we use Gaussian process regression to estimate age-specific effects (see above). The estimand is the predicted difference in probabilities averaged over all individuals in the target population. Therefore, in the second step, we calculate the age-specific differences between experimental conditions on the outcome scale (i.e. in terms of probability) and re-weight those estimates according to the frequency of age $k$ in the target population.

All models were fitted using Stan, a Hamiltonian Monte Carlo engine for Bayesian inference (Carpenter et al., 2017), implemented in R v.4.0.3 through Rstan v.2.21.2 (Stan Development Team, 2019). To reduce the risk of overfitting the data, we used weakly informative priors for all parameters. Visual inspection of traceplots and rank histograms (Vehtari et al., 2019) suggested good model convergence and no problematic autocorrelation, with convergence confirmed by the Gelman-Rubin criterion $\hat{R} \leq 1.01$ (Gelman et al., 1992). All inferences are based on over 1000 effective samples from the posterior (Gelman et al., 2013). The code necessary to reproduce all analyses is available on GitHub: https://github.com/DominikDeffner/Cross-Cultural-Generalizability. 


\section{B. Simulation example for demographic standardization}

The left-hand side of Figure S1 shows a simulated data example for the situation where populations differ in their demographic profile (Fig. 2a). Population I represents a growing population with many individuals in younger age classes, Population II, on the other hand, represents a shrinking population where most individuals are concentrated in older age classes (top row). Here, we assume that study samples are drawn randomly from the underlying populations, such that population differences are the sole causes of demographic disparities between samples. Older individuals are simulated to be more prosocial in their choices than younger individuals and women are more prosocial than men. There are also unobserved cultural factors causing higher prosociality in Population II (see top row of Fig. S2 for model estimates of age-/gender-specific probabilities to choose prosocial option in both populations). If our theoretical estimand is simply the mean trait value (e.g. the mean prosocial tendency) in each society, there is no need for poststratification and unadjusted estimates can be expected to recover the true population value (represented by dashed lines in bottom row of Fig. S1). This analysis reveals substantially larger prosociality in Population II compared to Population I. If we are not only interested in empirical population differences but in the influence of cultural norms or societal factors other than demography, we can compare values of the trait under comparable age and gender distributions. Here, we compute the counterfactual probabilities of choosing the prosocial option in Population I assuming the same demographic profile as Population II and vice versa. This analysis shows that in Population I there would still be lower overall prosociality compared to Population II, but the supposed "cultural difference" would be substantially smaller. Conversely, assuming the demography of Population I, individuals in Population II would still be more prosocial, but to a lesser extent. We could therefore conclude that the observed population differences in prosocial behavior are partly due to differences in the demographic characteristics and partly due to unobserved factors.

The right-hand side of Figure S1 shows a simulated example for the complementary situation where demographic profiles of the populations are identical but women are overrepresented in the sample from Population I and men are over-represented in the sample from Population II due to differences in local sampling procedures (Fig. 2b; see bottom row in Fig. S2 for gender- and age-specific estimates). In such a case, even the purely descriptive question of identifying the mean trait value in both populations requires poststratificationnaive empirical estimates will be systematically biased. Bottom row of Fig. S1 shows em-

pirical estimates as well as counterfactual estimates assuming the same demographic profile as the underlying population. This analysis shows that only the adjusted, poststratified, estimates accurately recover true population values. 
Population Differences

Sampling Differences
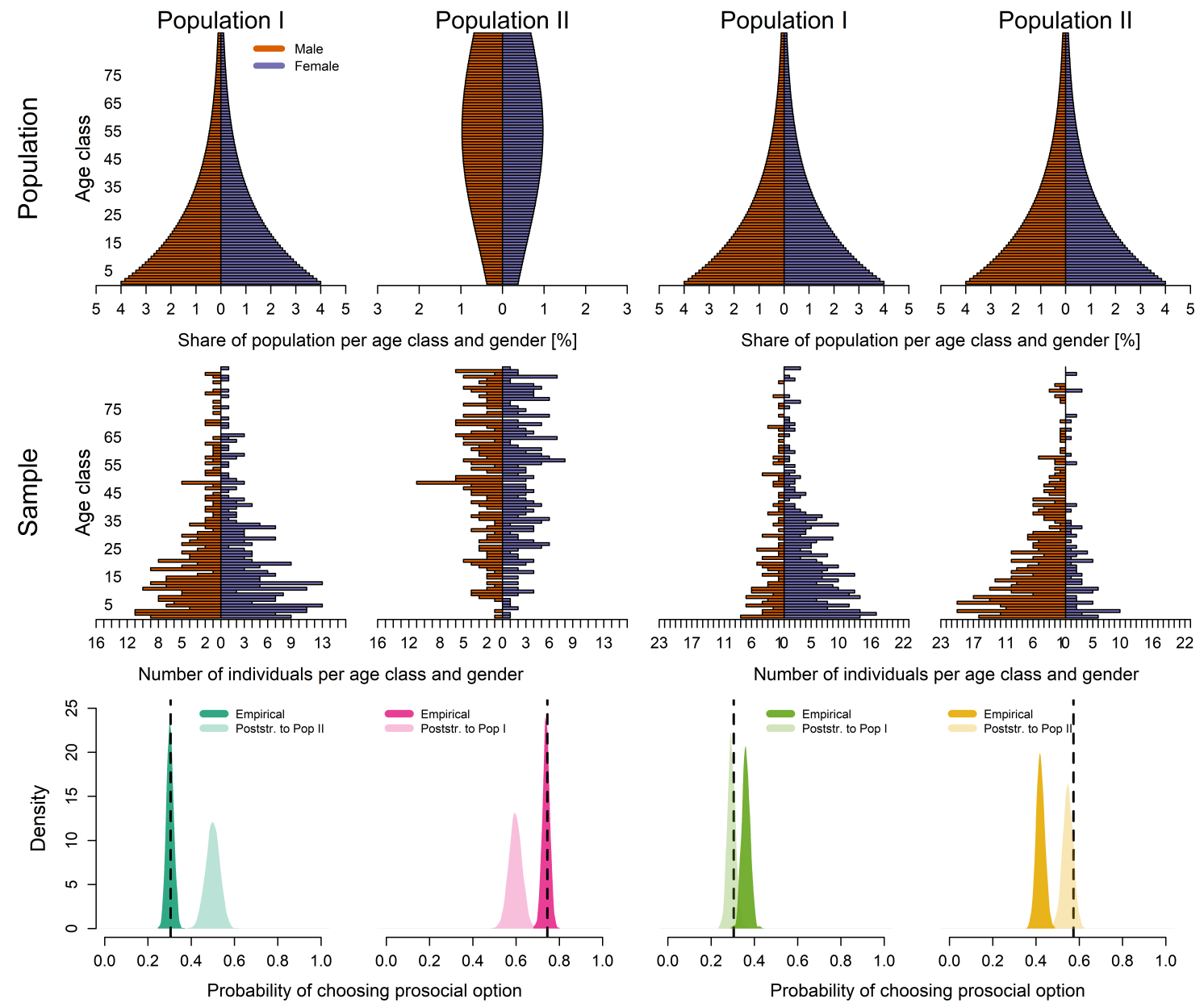

Figure S1. Simulation examples for demographic standardization. Population differences and counterfactual comparisons on the left (see Fig. 2a); adjustment for sampling differences on the right (see Fig. 2b). Top row shows demographic profiles of two populations, middle row shows demographic characteristics of $N=500$ study participants sampled from each population and bottom row shows estimates from multilevel regression with poststratification. For the scenario of population differences (left), poststratified estimates represent counterfactual comparisons assuming the same demographic profile as the other population; for the scenario of sampling differences (right), estimates are poststratified according to the demographic profile of the population from which samples are taken. Dashed lines represent true population means. 


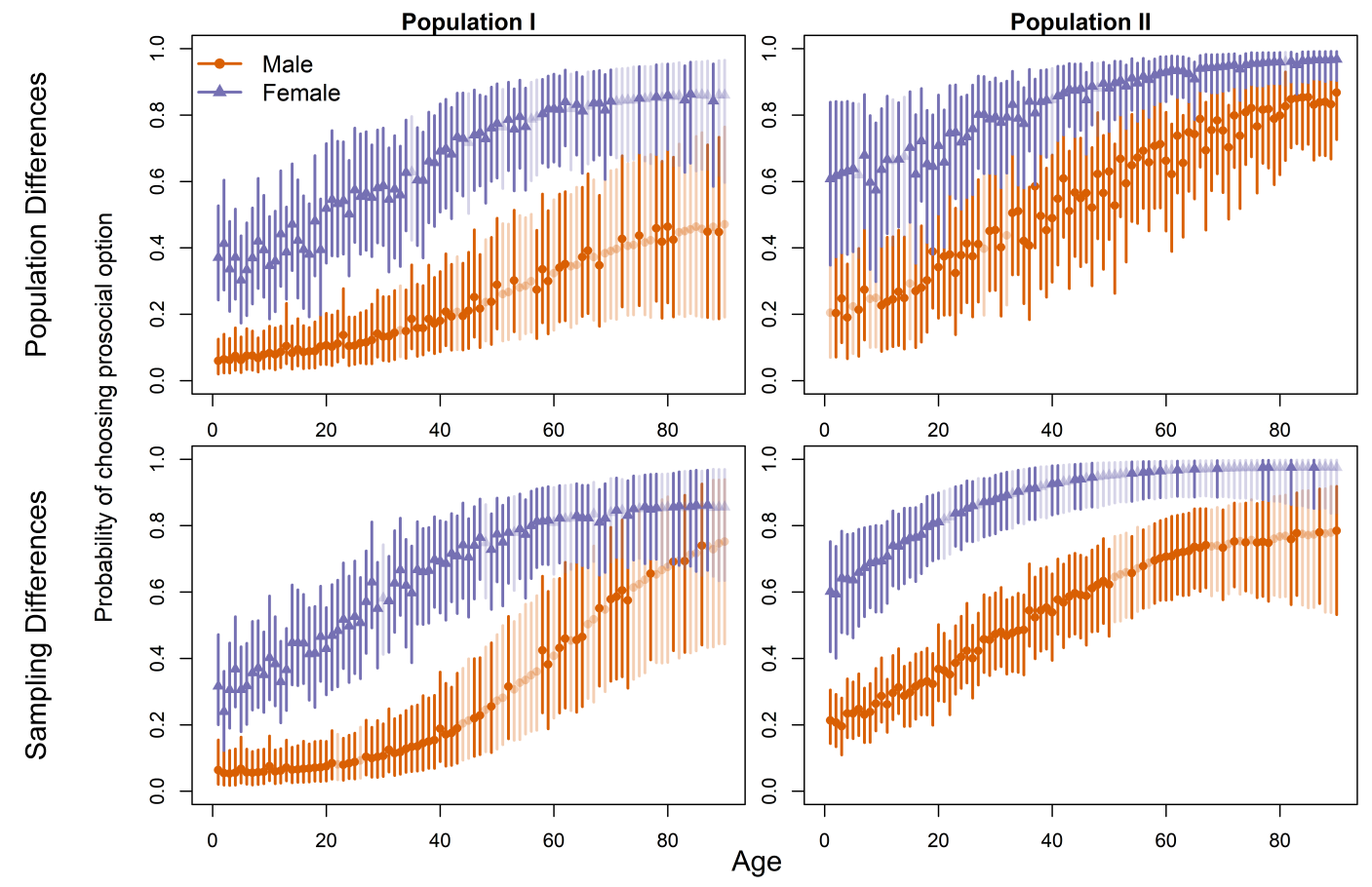

Figure S2. Demographic Standardization. Results of Gaussian process regression for simulation example. Probability of choosing prosocial option in two populations for individuals of different genders and ages. Results for population I are shown on the left, results for population II on the right. The top row shows results for scenario where populations differ in terms of their demographic profiles, the bottom row shows results for scenario where demographic disparities arise from different sampling strategies. Transparent shapes and lines show estimates for unobserved gender/age combinations.

\section{C. "S-admissibility" and transport procedures for proxy effect modifiers and differences in mechanism/mediator variables \\ S-admissibility}

A graphical criterion, called "S-admissibility" in the causal inference literature (Pearl and Bareinboim, 2014), lets us determine whether a $Z$-specific causal effect ( $Z$ represents set of covariates) observed in one population can be generalized to other populations. Sadmissibility states that the strata-specific causal effects are transportable if $Z$ d-separates (see Box 1) $Y$ from all selection nodes $S$ in the $X$-manipulated selection diagram. In other words, once all arrows pointing into the independent variable $X$ are removed, there needs to be a set of variables that can be conditioned on to shield the target quantity $Y$ from any source of disparity $S$. More intuitively, this means a causal effect is generalizable across populations if all relevant mechanisms by which populations are assumed to differ can be modelled.

Proxy effect modifiers

Suppose that we do not observe the true effect modifier, biological age, but only some proxy such as reported age $R$ and this proxy is observed to vary across populations (Figs. $4 \mathrm{c}, \mathrm{d}$ in the main text). This is actually a frequent problem in cross-cultural research as 
birth certificates are not available everywhere and societies might differ in the way ages are reported. Age heaping, for instance, describes the tendency of people familiar with the decimal system to round their age to the nearest 5 or 10 (Stockwell and Wicks, 1974; A'Hearn et al., 2009) and societies might also differ in terms of which ages are seen as more or less desirable. In this case, the correct procedure depends on the assumed causal mechanisms that generate disparities in $R$. If only the way ages are reported differs among societies while the true age distribution is the same (Fig. 4c), age can be ignored and experimental results can be directly generalized. If, on the other hand, the way ages are reported is the same across societies but populations differ in terms of average biological age (Fig. 4d), we can estimate age-specific effects as before and generalize using the age distribution of the target population. This again shows why generalizability is a causal, not a statistical, question. Both scenarios generate identical data distributions and the correct procedure depends solely on causal assumptions. Lastly, if ages are reported in a different way and there are also differences in the population age distribution, no generalizations are possible unless researchers are willing to make additional functional assumptions about the precise way ages are reported in different populations.

Differences in mechanism/mediator variables

Fig. 4e depicts a situation where there are no divergent effect modifiers but the mechanism mediating the effect of $X$ on $Y$ differs among societies. Imagine, for instance, that norm primes do not only directly affect behavior in the dictator game but also lead to the activation of internalized prosocial norms which then influences choices and this process of norm activation functions differently across societies. The procedure is more complicated, but there are now iterative, algorithmic solutions to determine how to generalize causal effects in the light of cultural differences in mediating mechanisms (Bareinboim and Pearl, 2012; Pearl and Bareinboim, 2014). In the example in Fig. 4e, generalization is possible because $N$ is S-admissible (i.e., it shields the outcome $Y$ from the sources of population disparity, see section above) and the effect of normative social information $X$ on norm activation $N$ is trivially transportable (i.e., it can be directly estimated in the target population without the need of experimental information). We can thus generalize the causal effect of $X$ on $Y$ by estimating strata-specific causal effects for each level of $N$ in the source population and adjust these probabilities according to the distribution of $N$ conditional on $X, P(n \mid x)$, in the target population. 


\section{Supplementary figures}

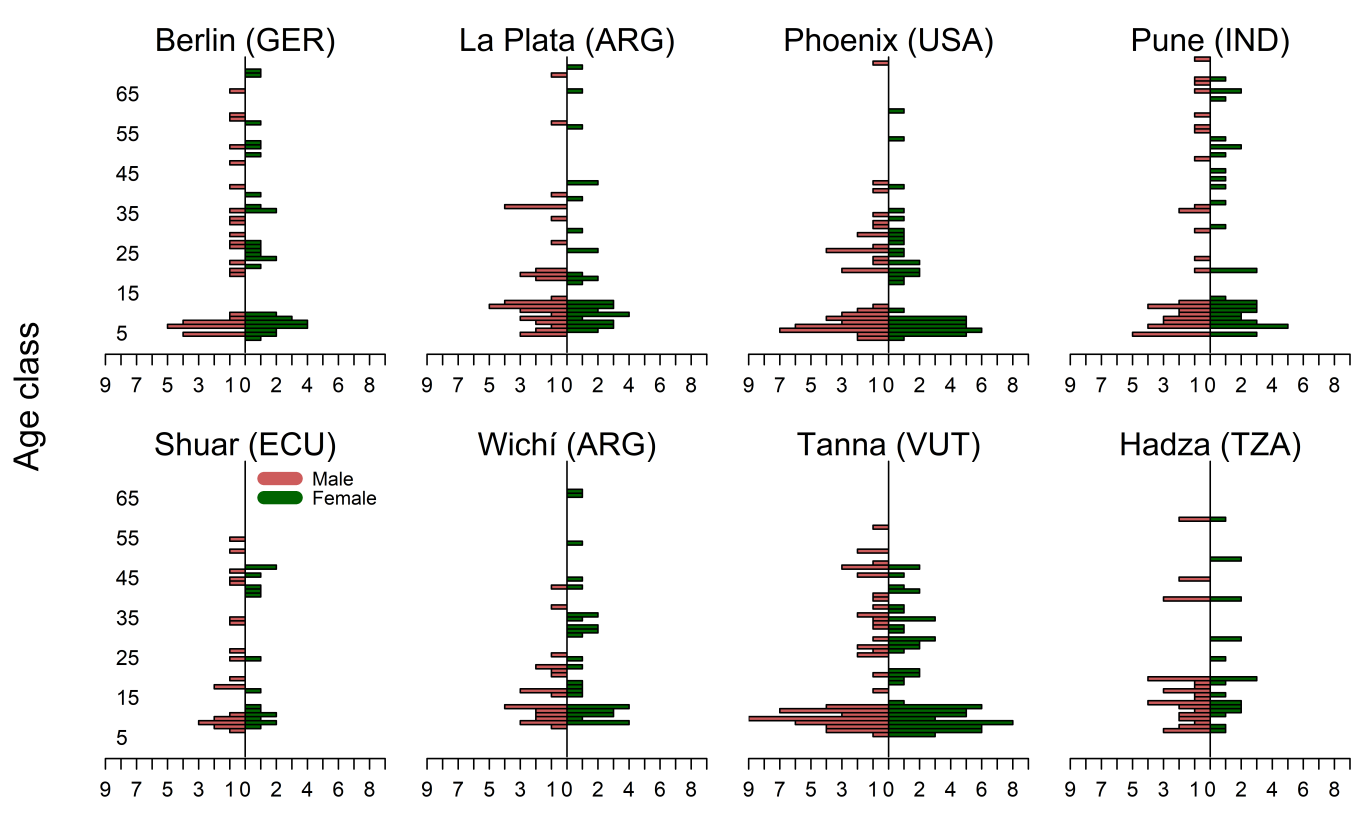

Number of individuals per age and gender

Figure S3. Demographic characteristics of study samples from 8 different populations included in House et al. (2020). For each population, bars represent the number of individuals per age class and gender (male in red, female in green).

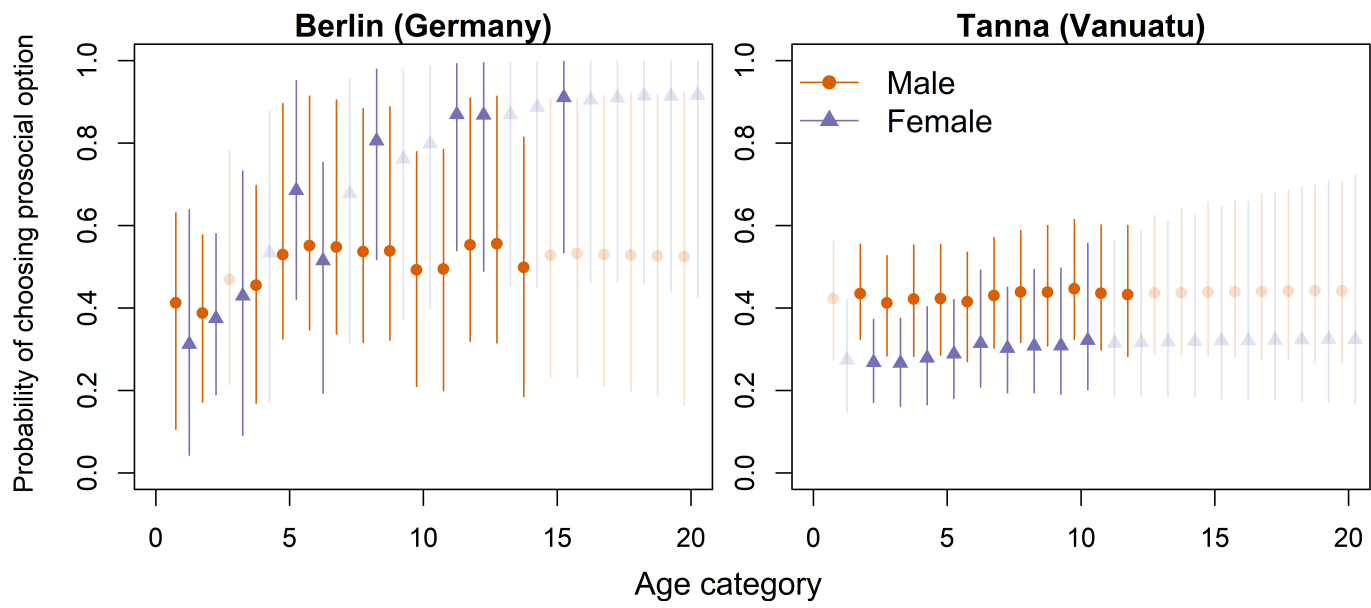

Figure S4. Demographic Standardization. Results of Gaussian process regression for real data example taken from House et al. (2020). Probability of choosing prosocial option in Berlin (Germany, left) and Tanna (Vanuatu, right) for individuals of different genders and age categories. Transparent shapes and lines show estimates for unobserved gender/age combinations. 


\section{REFERENCES}

A'Hearn, B., Baten, J., and Crayen, D. (2009). Quantifying quantitative literacy: Age heaping and the history of human capital. The Journal of Economic History, pages 783-808.

Bareinboim, E. and Pearl, J. (2012). Transportability of causal effects: Completeness results. In Proceedings of the AAAI Conference on Artificial Intelligence, volume 26.

Carpenter, B., Gelman, A., Hoffman, M. D., Lee, D., Goodrich, B., Betancourt, M., Brubaker, M., Guo, J., Li, P., and Riddell, A. (2017). Stan: A probabilistic programming language. Journal of statistical software, 76(1).

Gelman, A., Carlin, J. B., Stern, H. S., Dunson, D. B., Vehtari, A., and Rubin, D. B. (2013). Bayesian data analysis. Chapman and Hall/CRC.

Gelman, A. and Hill, J. (2006). Data analysis using regression and multilevel/hierarchical models. Cambridge university press.

Gelman, A., Rubin, D. B., et al. (1992). Inference from iterative simulation using multiple sequences. Statistical Science, 7(4):457-472.

House, B. R., Kanngiesser, P., Barrett, H. C., Broesch, T., Cebioglu, S., Crittenden, A. N., Erut, A., Lew-Levy, S., Sebastian-Enesco, C., Smith, A. M., et al. (2020). Universal norm psychology leads to societal diversity in prosocial behaviour and development. Nature Human Behaviour, 4(1):36-44.

McElreath, R. (2020). Statistical rethinking: A Bayesian course with examples in $R$ and Stan. CRC press.

Pearl, J. and Bareinboim, E. (2014). External validity: From do-calculus to transportability across populations. Statistical Science, pages 579-595.

Stan Development Team (2019). RStan: the R interface to Stan. R package version 2.19.2. Stockwell, E. G. and Wicks, J. W. (1974). Age heaping in recent national censuses. Social Biology, 21(2):163-167.

Vehtari, A., Gelman, A., Simpson, D., Carpenter, B., and Bürkner, P.-C. (2019). Ranknormalization, folding, and localization: An improved $\hat{R}$ for assessing convergence of memc. arXiv preprint arXiv:1903.08008. 